\title{
Article \\ Fractional-Order Edge Detection Masks for Diabetic Retinopathy Diagnosis as a Case Study
}

\author{
Samar M. Ismail ${ }^{1, *}$, Lobna A. Said ${ }^{2, *}$, , Ahmed H. Madian ${ }^{2,3}$ and Ahmed G. Radwan ${ }^{4,5, *}$ \\ 1 Faculty of Engineering and Technology, Egyptian Chinese University (ECU), Cairo 11787, Egypt \\ 2 Nanoelectronics Integrated Systems Center (NISC), Nile University, Giza 12588, Egypt; amadian@nu.edu.eg \\ 3 Radiation Engineering Department, NCRRT, Egyptian Atomic Energy Authority, Cairo 11829, Egypt \\ 4 Department of Engineering Mathematics and Physics, Cairo University, Giza 12613, Egypt \\ 5 School of Engineering and Applied Sciences, Nile University, Giza 12588, Egypt \\ * Correspondence: samar.ismail@guc.edu.eg (S.M.I.); 1.a.said@ieee.org (L.A.S.); agradwan@ieee.org (A.G.R.)
}

check for

updates

Citation: Ismail, S.M.; Said, L.A.; Madian, A.H.; Radwan, A.G. Fractional-Order Edge Detection Masks for Diabetic Retinopathy Diagnosis as a Case Study. Computers 2021, 10, 30. https://doi.org/ 10.3390/computers10030030

Received: 23 January 2021

Accepted: 25 February 2021

Published: 5 March 2021

Publisher's Note: MDPI stays neutral with regard to jurisdictional claims in published maps and institutional affiliations.

Copyright: (C) 2021 by the authors. Licensee MDPI, Basel, Switzerland. This article is an open access article distributed under the terms and conditions of the Creative Commons Attribution (CC BY) license (https:/ / creativecommons.org/licenses/by/ $4.0 /)$.

\begin{abstract}
Edge detection is one of the main steps in the image processing field, especially in biomedical imaging, to diagnose a disease or trace its progress. The transfer of medical images makes them more susceptible to quality degradation due to any imposed noise. Hence, the protection of this data against noise is a persistent need. The efficiency of fractional-order filters to detect fine details and their high noise robustness, unlike the integer-order filters, it renders them an attractive solution for biomedical edge detection. In this work, two novel central fractional-order masks are proposed with their detailed mathematical proofs. The fractional-order parameter gives an extra degree of freedom in designing different masks. The noise performance of the proposed masks is evaluated upon applying Salt and Pepper noise and Gaussian noise. Numerical results proved that the proposed masks outperform the integer-order masks regarding both types of noise, achieving higher Peak Signal to Noise Ratio. As a practical application, the proposed fractional-order edge detection masks are employed to enhance the Diabetic Retinopathy disease diagnosis.
\end{abstract}

Keywords: edge detection; fractional-order; noise; diabetic retinopathy

\section{Introduction}

Nowadays, digital imaging has invaded every aspect of our lives, starting from personal to professional perspectives, including data saving or transferring data through the internet. The effect of some additive noise may distort data through transmission, which may cause erroneous results after its retrieval, whether data analysis was performed for security purposes or medical diagnosis applications or any other purpose. Preserving data against noise distortion is one of the main concerns in data handling through transmission, where some image processing steps should be considered to eliminate or even reduce such losses. Edge detection is mainly one of these steps in digital image preprocessing, pattern recognition, and biomedical image analysis; hence, it is still an active research area. By definition, edges are the sudden changes in the intensity of image pixels. They take place at the boundary of various intensity regions in the image [1]. Edge detection is performed using gradient masks classified into two main categories: first-order derivative masks and second-order derivative masks. The edges are inspected by detecting the peaks using first-order derivative operators such as Sobel or Prewitt or Roberts operators, or by detecting the zero crossings using second-order derivative operators like Laplacian operator. These operators are seen as filters, or masks convoluted with the original image under test to obtain the binary edge detected image, sometimes called "edge map", thus, came the term "convolution masks" [2].

The most widely used integer first-order derivative operators are Sobel operator, Roberts operator and Prewitt operator, shown in Table 1. These are the classical gradientbased edge detection filters. They detect the horizontal and vertical edges individually 
based on a pair of convolution operators. In Sobel's case for example, the operator is applied separately to a center point pixel of $3 \times 3$ neighbourhood, where some smoothing can be achieved by using a weight value of 2 to give more importance to the center point [2] In Table 1, the filters are applied to "Lena" image, and the corresponding edge-detected images are presented.

Table 1. Firstorder derivative edge detection operators.

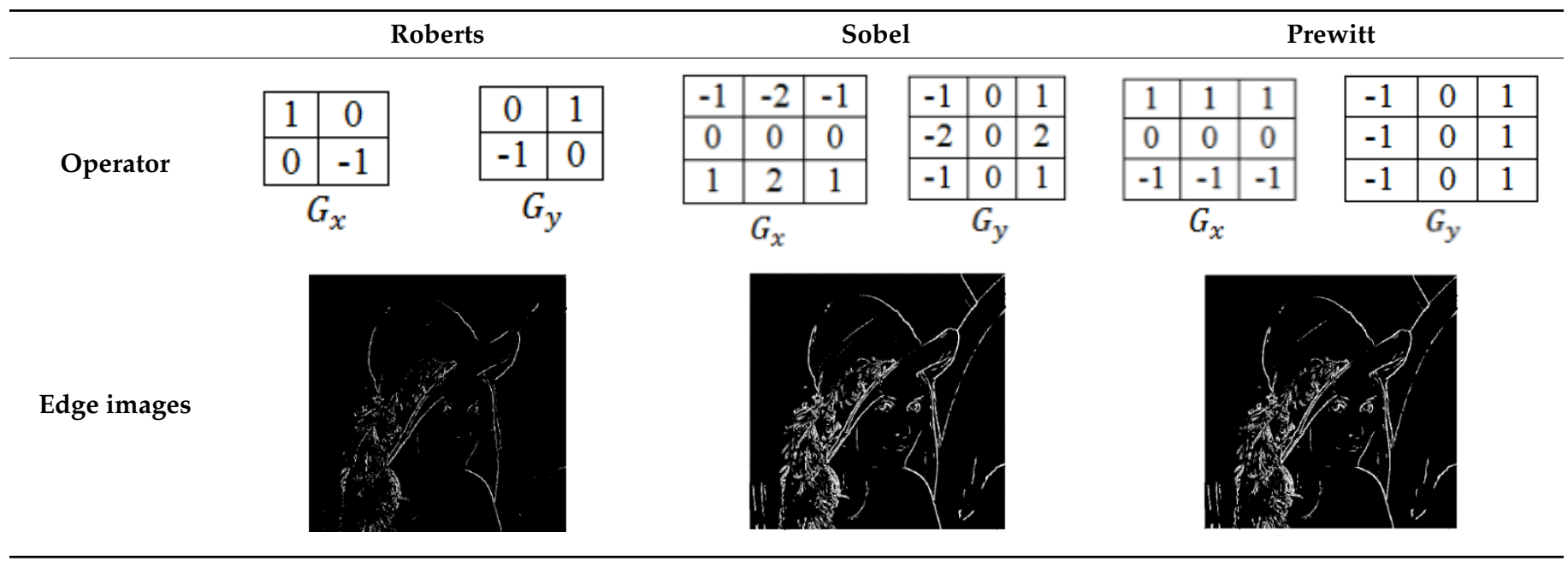

Mathematically, for a continuous two-dimensional function $f$, the integer order first derivative is defined by the vector:

$$
\nabla f=\left[\begin{array}{l}
G_{x} \\
G_{y}
\end{array}\right]=\left[\begin{array}{ll}
\frac{\partial f}{\partial x} & \frac{\partial f}{\partial y}
\end{array}\right]^{T} .
$$

The magnitude of the gradient is given by

$$
\operatorname{mag}(\nabla f)=\sqrt{G_{x}^{2}+G_{y}^{2}}=\sqrt{\left(\frac{\partial f}{\partial x}\right)^{2}+\left(\frac{\partial f}{\partial y}\right)^{2}} .
$$

As an approximation, the magnitude of the gradient can be considered using the absolutes:

$$
G[f(x, y)] \approx\left|G_{x}\right|+\left|G_{y}\right| .
$$

Considering digital images, the derivatives are approximated by differences. Then the gradient is calculated as

$$
G_{x} \cong f[x+1, y]-f[x, y], G_{y} \cong f[x, y+1]-f[x, y] .
$$

The Fractional Calculus (FC) is thought of as the generalization of the conventional calculus dealing with non-integer orders for integration and differentiation. Fractionalorder Integral/Differential operators have a continuous manner, unlike the classical integerorder integration/differentiation, which is just a special case of the FC [3]. Scientists started to wonder about the existence of fractional-order derivatives at the end of the seventeenth century. However, fractional-order theories did not cross the boundary of pure theoretical mathematics to real applications until recently, when researchers discovered the strength of this mathematical branch in explaining many complex phenomena [4]. Since then, FC has contributed to nearly every research field [5], such as in electronics design, where FC was used in the electrical characterization of supercapacitors in [6], and in [7] the design of oscillators was based on fractional-order capacitors, while in [8] the Field-Programmable Gate Array (FPGA) implementation of fractional order image edge detector was provided. 
FC also shared in bioengineering [9], encryption [10], and chaos theory [11,12], as well as, in biomedical image processing like Alzheimer disease detection in [13] and Brain Tumor Detection in [14] using fractional order edge detectors. The fractional order derivative can be performed using different operators, based on the definitions of Caputo and GrunwaldLetnikov's (GL) [3]. Nevertheless, the GL definition was found to be easily applied in digital image processing applications than the Caputo one. An extra degree of freedom is added to any design by including the fractional-order parameter, which increases the flexibility and controllability of the system.

Similar to Equation (1), for a continuous two-dimensional function $f$, the fractionalorder derivative operator is defined as follows by the vector:

$$
\nabla^{\alpha} f=\left[\begin{array}{l}
G_{x} \\
G_{y}
\end{array}\right]=\left[\begin{array}{ll}
\frac{\partial^{\alpha} f}{\partial x^{\alpha}} & \frac{\partial^{\alpha} f}{\partial y^{\alpha}}
\end{array}\right]^{T},
$$

where $\alpha$ is the fractional-order parameter.

In digital images, for the ease of analysis, implementation and faster computation, the magnitude of the fractional-order gradient will be also computed using the absolutes as an approximation:

$$
\operatorname{mag}\left(\nabla^{\alpha} f\right)=\left|G_{x}\right|+\left|G_{y}\right| .
$$

Based on the target of the image processing step, one can choose between the first-order or the second-order derivative methods. Generally, the first-order derivative operators produce thicker edges than the second-order ones, resulting in the loss of some image details. In some applications, especially in biomedical applications, such as disease diagnosis or disease progress follow up, this choice could be a disaster missing the crucial details in the images under test. The second-order derivative methods are more efficient in detecting the fine details, yet they are more noise sensitive, and in this era of data transfer, images may be susceptible to some noise degradation. Hence, one cannot neglect this issue also in applying the second-order masks. Thus, it has been ever a trade-off aspect to decide whether to use the first-order operators with their sufficient noise robustness at the expense of producing thick edges and missing some fine details in the detected images; or to use the second-order operators, with their high capability in detecting fine details at the expense of their noise sensitivity [2]. Upon employing fractional-order edge detection operators, this conflict has been significantly resolved to retain high noise robustness and still detect fine details. This is achieved by targeting specific frequencies by tuning the fractional-order parameter. This offers the designer higher degrees of freedom while choosing the fractionalorder mask. On comparing the fractional differential operators with the integer-order ones, it was found that the first outperforms the second in the image processing field [15]. The reason behind this is that the fractional order derivative consists of infinite number of terms, exhibiting a memory effect retaining more image details than the integer-order ones, where the integer is just one special case of the general fractional-order derivative when $\alpha=1$.

This work aims to propose two novel central continuous fractional-order gradient masks to be employed for image edge detection. The mathematical proofs of the proposed masks are presented. As a proof of concept of the novel fractional masks, the masks' noise robustness is tested against Gaussian noise distortion and Salt and Pepper noise distortion for different sets of images. As an application, the proposed fractional-order edge detection masks are employed to aid in the diagnosis of Diabetic Retinopathy patients based on the superiority of fractional-order masks in detecting more delicate details of noisy images rather than the integer-order conventional ones.

The paper is organized as follows: Section 2 provides a survey on recent work of fractional-order edge detection in literature, followed by the novel fractional-order masks proposed in Section 3. Simulation results of the proposed masks in edge detection are presented in Section 4, discussing in detail the robustness of the proposed masks against different types of noise. Section 5 presents the Diabetic Retinopathy (DR) image diagnosis 
as an application of the fractional-order edge detection employing the superiority of the proposed masks against noise distortion, and finally, Section 6 concludes this work.

\section{Survey on Fractional-Order Image Edge Detection}

As previously mentioned in the introduction, thicker edges are obtained when firstorder derivative operators are employed, rather than the second-order ones, resulting in losing some of the image details. The second-order derivative methods should be used to detect the delicate details efficiently, on the expense of being more noise sensitive. Fractional-order derivative masks have appeared lately to solve this conflict or trade-off crucially. Edge detection methods are greatly enhanced upon using fractional-order masks, regarding noise sensitivity. They also help in controlling some features in the image by targeting specific frequency components in the image.

Hereby, a fractional-order edge detection survey is presented, elaborating how FC has contributed in many image processing applications in recent decades. In [15], the fractional differential approach was employed developing three fractional-order edge detection masks; these Fractional Masks (FM) are shown in Table 2. In [16], the combination of fractional-order edge detection and a chaos synchronization classifier was proposed to be employed in fingerprint identification. To overcome the limitations of the integral-order way, following the GL definition, a fractional-order edge detection mask was proposed to obtain clearer ridge and valley structures in the fingerprint images. The employed mask in [16] is shown in Table 2 in both the $x$ - and y-directions. A Fractional Differential Algorithm was used for Palm Print edge extraction in [17], while in [18], numerical experiments showed that fractional-order masks have higher capabilities as compared to the integer-order ones in enhancing the texture details of rich-grained digital images. In [19], the fractional approach was applied on an RGB bank slip image, where it was observed that the complex textural information of the original bank slip image became clearer after implementing the fractional differential approach. At the same time, it was lost after implementing the integral differential. Another application of the fractional-order differential calculus was in astronomical image analysis introduced in [20]. A processing tool was developed to enhance the quality of astronomical images. It was concluded that using the fractional-order gradient masks helped in detecting faint objects and galaxy structures as well as surface details enhancement.

In [21], a combination of two different fractional-order derivatives is used for smoothing different image regions and noise removal. A novel edge detector was also introduced which was superior in dealing with repetitive structures and textures in an image as well as eliminating noise. In [22], a model is proposed for developing a texture detection algorithm inspired by edge detection algorithms, assuming that the texture features are similar to edges, hence reducing the time complexity. Fractional partial derivatives, Gaussian Kernel smoothing as well as a statistical approach was employed in the proposed model. Different types of textured images were used for validation, where high classification accuracy was achieved.

In [23], several fractional masks were presented and applied for image denoising. These masks were based on the fractional integration method. The first used the GL scheme called (GL-ABC), while the second used the Toufik-Atangana scheme (TA-ABC), and the third one used the fractional Euler method (Eu-ABC). The process constructed a square and then applied it to all the blocks in the noisy image. The results showed that the proposed masks are efficient, and their performances are compatible with other fractional smoothing filters. In [24], a fractional-order edge detector was applied to a real application to detect edges in a railway track measurement system, for improving the detection performance of noisy images significantly. A new edge detection operator is realized in [25], upon combining fractional differentiation and integration which resulted in a complex mask; such mask was efficient in noise suppression while detecting edges of noisy images with high accuracy as compared with Canny operator. Another application of the fractional differential theory was also highlighted upon improving the fractional differential Tiansi 
operator, to enhance the edge detection ability of fractional order masks. The improved masks showed more effective performance than the traditional operators, especially with images with fine edges as rock fracture images as presented in [26]. In [27], a fractional edge detection convolution mask was constructed based on the Riemann-Liouville fractional derivative, which is a special form of the Srivastava-Owa operator. The resultant mask was able to trap both local intensity discontinuities, as well as, locate Dirac edges. Another application for fractional-order masks was in road obstacle detection in [28], where it was found that fractional-order masks yielded more precise edges, which is a desirable result allowing better obstacle detection. Moreover, a generalized fractional-order Sobel operator was introduced in [29], presented in Table 2, for structure feature extraction of medical images.

Some researches were applied for image contrast enhancement using fractional order masks in [30]. For instance, based on GL definition, a fractional-order mask was presented, and when followed by a Sobel edge detector, the algorithm was able to detect the details of low contrast images with better sharpness and higher accuracy than the traditional integerorder masks [31]. The proposed algorithm in such work enhanced the high-frequency components in the images while preserving the low-frequency ones. Moreover, RiemannLiouville fractional differential operator was utilized to implement feature and contrast enhancement of images in [32].

In addition to all the previous applications, the fractional-order edge detection masks exhibited a higher hand in the medical imaging field in the literature [33]. The image processing of medical images in the fractional order domain was also tackled in [34], while in [35], a genetic algorithm was used to optimize the fractional order edge detection to diagnose Alzheimer disease (AD). Another method to detect AD was also presented in [36] based on fractional-order edge detection of Magnetic Resonance Imaging (MRI) images. In [37], a new fractional-order mask was introduced for edge detection and was applied on tracing breast cancer mammograms images. Moreover, a robust image corner detector based on a fractional gradient operator was proposed in [38], where the gradient operator was derived using fractional-order differentiation and integration, and further applied on MRI images for validation. Another edge detection technique based on Riesz fractional derivative in the Fractional Fourier Transform domain was proposed in [39] and then used for image enhancement. An edge-based Active contour segmentation model was presented in [40], where the edge-based term was derived using Caputo-Fabrizio based fractional-order Gaussian derivatives and then applied to compute the weighted area of the region inside the contour.

An algorithm based on fractional differential GL definition was also presented in [17]; three filter templates were constructed to extract the thenar palm print edge; one of these masks is shown in Table 2. The proposed algorithm was proved to be capable of noise reduction as well as detecting rich edge details of images than traditional methods. Last but not least, another application of fractional-order edge detection was introduced in [10], where it was combined with chaotic pseudorandom number generators in an image encryption system; which system gave comparable results with other existing complex image encryption schemes.

In this work, two novel fractional-order masks are proposed, where the mathematical derivations of these masks are presented in detail in the next section. 
Table 2. Some fractional-order masks of previous work in literature.

\begin{tabular}{|c|c|c|c|c|c|c|c|c|}
\hline \multirow{4}{*}{ 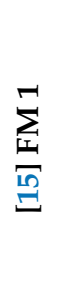 } & \multirow{4}{*}{$G_{x}$} & \multicolumn{2}{|l|}{0} & 0 & \multirow{4}{*}{$G_{y}$} & $(\alpha$ & \multirow{2}{*}{$\frac{\left(\alpha^{2}-\alpha\right)}{2}$} & \multirow{2}{*}{0} \\
\hline & & $\left(\alpha^{2}-\alpha\right)$ & \multirow{2}{*}{$-\alpha$} & \multirow{2}{*}{1} & & & & \\
\hline & & 2 & & & & 0 & $-\alpha$ & 0 \\
\hline & & 0 & 0 & 0 & & 0 & 1 & 0 \\
\hline \multirow{3}{*}{$\frac{\underset{|c|}{N}}{\stackrel{N}{\Xi}}$} & \multirow{3}{*}{$G_{x}$} & $\frac{\alpha^{2}-\alpha}{6}$ & $\frac{\alpha^{2}-\alpha}{6}$ & $\frac{\alpha^{2}-\alpha}{6}$ & \multirow{3}{*}{$G_{y}$} & $\frac{\alpha^{2}-\alpha}{6}$ & $\frac{-\alpha}{5}$ & $\frac{-\alpha}{5}$ \\
\hline & & $\frac{-\alpha}{5}$ & $\frac{-\alpha}{5}$ & $\frac{-\alpha}{5}$ & & $\frac{\alpha^{2}-\alpha}{6}$ & $\frac{-\alpha}{5}$ & 1 \\
\hline & & $\frac{-\alpha}{5}$ & 1 & $\frac{-\alpha}{5}$ & & $\frac{\alpha^{2}-\alpha}{6}$ & $\frac{-\alpha}{5}$ & $\frac{-\alpha}{5}$ \\
\hline \multirow{4}{*}{$\frac{\sum_{i=}^{\infty}}{\stackrel{\infty}{\Xi}}$} & \multirow{4}{*}{$G_{x}$} & $\mathbf{0}$ & $\mathbf{0}$ & 1 & \multirow{4}{*}{$G_{y}$} & $\underline{\alpha^{2}-\alpha}$ & 0 & 0 \\
\hline & & & & 0 & & 2 & & \\
\hline & & & & & & 0 & $-\alpha$ & $\mathbf{0}$ \\
\hline & & $\frac{\alpha^{2}-\alpha}{2}$ & 0 & 0 & & 0 & 0 & 1 \\
\hline \multirow{3}{*}{$\stackrel{\Xi}{\Xi}$} & \multirow{3}{*}{$G_{x}$} & 0 & $\frac{\alpha^{2}-\alpha}{2}$ & 0 & \multirow{3}{*}{$G_{y}$} & 0 & 0 & 0 \\
\hline & & 0 & $-\alpha$ & 0 & & $\frac{\alpha^{2}-\alpha}{2}$ & $-\alpha$ & 1 \\
\hline & & $\mathbf{0}$ & 1 & 0 & & 0 & 0 & 0 \\
\hline \multirow{3}{*}{$\Xi$} & $-\alpha$ & $-\alpha$ & $-\alpha$ & \multirow{3}{*}{$\begin{array}{l}\bar{\Xi} \\
\stackrel{0}{0} \\
0 \\
0 \\
\text { 焉 }\end{array}$} & & $\frac{\alpha^{2}-\alpha}{4}$ & $\frac{\alpha^{2}-\alpha}{2}$ & $\frac{\alpha^{2}-\alpha}{4}$ \\
\hline & $-\alpha$ & 8 & $-\alpha$ & & & $\frac{-\alpha}{2}$ & $-\alpha$ & $\frac{-\alpha}{2}$ \\
\hline & $-\alpha$ & $-\alpha$ & $-\alpha$ & & & $\frac{1}{2}$ & 1 & $\frac{1}{2}$ \\
\hline
\end{tabular}




\section{Proposed Fractional-Order Sobel Mask}

The classical integer-order Sobel mask is presented in Table 1, with its gradient components in both the $\mathrm{x}$ - and $\mathrm{y}$ - directions. The mathematical differential form of these components can be expressed as follows:

$$
\begin{aligned}
& G_{x}=2\left(\frac{\partial f(x+1, y-1)}{\partial x}+2 \frac{\partial f(x+1, y)}{\partial x}+\frac{\partial f(x+1, y+1)}{\partial x}\right), \\
& G_{y}=2\left(\frac{\partial f(x-1, y+1)}{\partial y}+2 \frac{\partial f(x, y+1)}{\partial y}+\frac{\partial f(x+1, y+1)}{\partial y}\right) .
\end{aligned}
$$

The representation of the $G_{x}$ and $G_{y}$ components of the original Sobel filter can be modified as shown in Figure 1. In this section, the modified Sobel filter is generalized to the fractional-order domain, using the differential form of the gradient operator in Equation (7), following the GL fractional-order differ-integral operator, to obtain two novel masks named Left-Sided Fractional-Order Mask (LS-FOM) and the Right-Sided Fractional-Order Mask (RS-FOM).

\begin{tabular}{c|c|c|c|} 
& 0 & 0 & 0 \\
\hline $\boldsymbol{G}_{\boldsymbol{x}}$ & -1 & -2 & -1 \\
\hline & 1 & 2 & 1 \\
\hline
\end{tabular}

\begin{tabular}{|c|c|c|c|} 
& -1 & 1 & 0 \\
\hline $\boldsymbol{G}$ & -2 & 2 & 0 \\
\hline & -1 & 1 & 0 \\
\hline
\end{tabular}

\begin{tabular}{|c|c|c|}
\hline-1 & -2 & -1 \\
\hline 1 & 2 & 1 \\
\hline 0 & 0 & 0 \\
\hline
\end{tabular}

\begin{tabular}{|l|l|l|}
\hline 0 & -1 & 1 \\
\hline 0 & -2 & 2 \\
\hline 0 & -1 & 1 \\
\hline
\end{tabular}

\begin{tabular}{|c|c|c|}
\hline-1 & -2 & -1 \\
\hline 0 & 0 & 0 \\
\hline 1 & 2 & 1 \\
\hline
\end{tabular}

\begin{tabular}{|l|l|l|}
\hline-1 & 0 & 1 \\
\hline-2 & 0 & 2 \\
\hline-1 & 0 & 1 \\
\hline
\end{tabular}

(a)

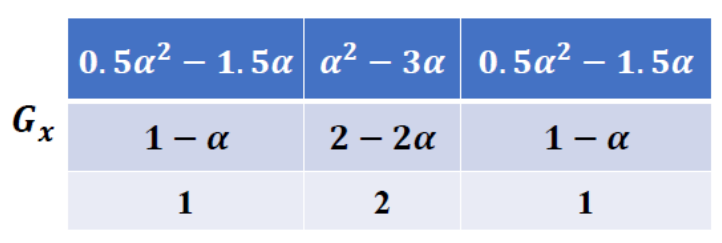

(b)

\begin{tabular}{|c|c|c|c|}
\hline \multirow{3}{*}{$G_{y}$} & $0.5 \alpha^{2}-1.5 \alpha$ & $1-\alpha$ & 1 \\
\hline & $\alpha^{2}-3 \alpha$ & $2-2 \alpha$ & 2 \\
\hline $0.5 \alpha^{2}-1.5 \alpha$ & $1-\alpha$ & 1 \\
\hline
\end{tabular}

\begin{tabular}{|c|c|c|c|}
\hline & -1 & $\alpha-1$ & $1.5 \alpha-0.5 \alpha^{2}$ \\
\hline$G_{y}$ & -2 & $2 \alpha-2$ & $3 \alpha-\alpha^{2}$ \\
\hline & -1 & $\alpha-1$ & $1.5 \alpha-0.5 \alpha^{2}$
\end{tabular}

(c)

Figure 1. (a) The modified Sobel Representation, (b) Left-sided fractional-order Sobel, and (c) Right-sided fractionalorder Sobel.

\subsection{Left-Sided Fractional-Order Mask (LS-FOM)}

The left-sided GL derivatives can be defined as follows [41]:

$$
\operatorname{mag}\left(\nabla^{\alpha} f\right) D_{t}^{\alpha} f(t)=\lim _{h \rightarrow 0^{+}} \frac{1}{h^{\alpha}} \sum_{j=0}^{\infty}(-1)^{j}\left(\begin{array}{c}
\alpha \\
j
\end{array}\right) f(t-j h),=\left|G_{x}\right|+\left|G_{y}\right| .
$$


To assure that a symmetric filter is obtained, the GL operator in Equation (8) is applied on the modified Sobel filter representation. The fractional-order left-sided differential form of the gradient components along the $\mathrm{x}$ - and $\mathrm{y}$ - directions can be written as follows:

$$
\begin{aligned}
& G_{x}=\frac{\partial^{\alpha} f(x+1, y-1)}{\partial x^{\alpha}}+2 \frac{\partial^{\alpha} f(x+1, y)}{\partial x^{\alpha}}+\frac{\partial^{\alpha} f(x+1, y+1)}{\partial x^{\alpha}}+\frac{\partial^{\alpha} f(x, y-1)}{\partial x^{\alpha}}+2 \frac{\partial^{\alpha} f(x, y)}{\partial x^{\alpha}}+\frac{\partial^{\alpha} f(x, y+1)}{\partial x^{\alpha}}, \\
& G_{y}=\frac{\partial^{\alpha} f(x-1, y)}{\partial y^{\alpha}}+2 \frac{\partial^{\alpha} f(x, y)}{\partial y^{\alpha}}+\frac{\partial^{\alpha} f(x+1, y)}{\partial y^{\alpha}}+\frac{\partial^{\alpha} f(x-1, y+1)}{\partial y^{\alpha}}+2 \frac{\partial^{\alpha} f(x, y+1)}{\partial y^{\alpha}}+\frac{\partial^{\alpha} f(x+1, y+1)}{\partial y^{\alpha}} .
\end{aligned}
$$

Using the left-sided GL derivative, Equation (9) could be approximated as follows:

$$
\begin{aligned}
G_{x} & =f(x+1, y-1)-\alpha f(x, y-1)+\frac{(\alpha)(\alpha-1)}{2} f(x-1, y-1)+\ldots \frac{(-1)^{n} \Gamma(\alpha+1)}{n ! \Gamma(\alpha-n+1)} f(x+1-n, y-1) \\
& +2 f(x+1, y)-2 \alpha f(x, y)-(\alpha)(\alpha-1) f(x-1, y)+\ldots \frac{2(-1)^{n} \Gamma(\alpha+1)}{n ! \Gamma(\alpha-n+1)} f(x+1-n, y) \\
& +f(x+1, y+1)-\alpha f(x, y+1)+\frac{(\alpha)(\alpha-1)}{2} f(x-1, y+1) \\
& +\ldots \frac{(-1)^{n} \Gamma(\alpha+1)}{n ! \Gamma(\alpha-n+1)} f(x+1-n, y+1)+f(x, y-1)-\alpha f(x-1, y-1) \\
& +\ldots \frac{(-1)^{n} \Gamma(\alpha+1)}{n ! \Gamma(\alpha-n+1)} f(x-n, y-1)+2 f(x, y)-2 \alpha f(x-1, y) \\
& +\ldots \frac{2(-1)^{n} \Gamma(\alpha+1)}{n ! \Gamma(\alpha-n+1)} f(x-n, y)+f(x, y+1)-\alpha f(x-1, y+1) \\
& +\ldots \frac{(-1)^{n} \Gamma(\alpha+1)}{n ! \Gamma(\alpha-n+1)} f(x-n, y+1), \\
G_{y} & =f(x-1, y)-\alpha f(x-1, y-1)+\frac{(\alpha)(\alpha-1)}{2} f(x-1, y-2)+\ldots \frac{(-1)^{n} \Gamma(\alpha+1)}{n ! \Gamma(\alpha-n+1)} f(x-1, y-n)++2 f(x, y) \\
& -2 \alpha f(x, y-1)+\ldots \frac{2(-1)^{n} \Gamma(\alpha+1)}{n ! \Gamma(\alpha-n+1)} F(x, y-n)+f(x+1, y) \\
& -\alpha f(x+1, y-1)+\ldots \frac{(-1)^{n} \Gamma(\alpha+1)}{n ! \Gamma(\alpha-n+1)} f(x+1, y-n)+f(x-1, y+1)-\alpha f(x-1, y) \\
& +\frac{(\alpha)(\alpha-1)}{2} f(x-1, y-1)+\ldots \frac{(-1)^{n} \Gamma(\alpha+1)}{n ! \Gamma(\alpha-n+1)} f(x-1, y+1-n)+2 f(x, y+1)-2 \alpha f(x, y) \\
& +(\alpha)(\alpha-1) F(x, y-1) \ldots \frac{2(-1)^{n} \Gamma(\alpha+1)}{n ! \Gamma(\alpha-n+1)} f(x, y+1-n)+f(x+1, y+1)-\alpha f(x+1, y) \\
& +\frac{(\alpha)(\alpha-1)}{2} f(x+1, y-1) \ldots \frac{(-1)^{n} \Gamma(\alpha+1)}{n ! \Gamma(\alpha-n+1)} f(x+1, y+1-n) .
\end{aligned}
$$

\subsection{Right-Sided Fractional-Order Mask (RS-FOM)}

The right-sided GL derivatives can be defined as follows [41]:

$$
D_{t}^{\alpha} f(t)=\lim _{h \rightarrow 0^{+}} \frac{1}{h^{\alpha}} \sum_{j=0}^{\infty}(-1)^{j}\left(\begin{array}{c}
\alpha \\
j
\end{array}\right) f(t+j h) .
$$

The fractional-order right-sided differential form of the gradient components of the modified Sobel filter representation shown in Figure 1 along the $x$ - and $y$-directions can be written as follows:

$$
\begin{gathered}
G_{x}=-\frac{\partial^{\alpha} f(x, y-1)}{\partial x^{\alpha}}-2 \frac{\partial^{\alpha} f(x, y)}{\partial x^{\alpha}}-\frac{\partial^{\alpha} f(x, y+1)}{\partial x^{\alpha}}-\frac{\partial^{\alpha} f(x-1, y-1)}{\partial x^{\alpha}}-2 \frac{\partial^{\alpha} f(x-1, y)}{\partial x^{\alpha}}-\frac{\partial^{\alpha} f(x-1, y+1)}{\partial x^{\alpha}}, \\
G_{y}=-\frac{\partial f(x-1, y-1)}{\partial y}-2 \frac{\partial f(x, y-1)}{\partial y}-\frac{\partial f(x+1, y-1)}{\partial y}-\frac{\partial f(x-1, y)}{\partial y}-2 \frac{\partial f(x, y)}{\partial y}-\frac{\partial f(x+1, y)}{\partial y} .
\end{gathered}
$$

Using the right-sided GL derivative, Equation (12) could be approximated as follows: 


$$
\begin{aligned}
& G_{x}=-f(x, y-1)+\alpha f(x+1, y-1)+\ldots \frac{(-1)^{n+1} \Gamma(\alpha+1)}{n ! \Gamma(\alpha-n+1)} f(x+n, y-1)-2 f(x, y)+2 \alpha f(x+1, y) \\
&+\ldots \frac{2(-1)^{n+1} \Gamma(\alpha+1)}{n ! \Gamma(\alpha-n+1)} f(x+n, y-1)-f(x, y+1)+\alpha f(x+1, y+1) \\
&+\ldots \frac{(-1)^{n+1} \Gamma(\alpha+1)}{n ! \Gamma(\alpha-n+1)} f(x+n, y-1)-f(x-1, y-1)+\alpha f(x, y-1) \\
& \quad-\frac{(\alpha)(\alpha-1)}{2} f(x+1, y-1)+\ldots \frac{(-1)^{n+1} \Gamma(\alpha+1)}{n ! \Gamma(\alpha-n+1)} f(x-1+n, y-1)-2 f(x-1, y) \\
&+2 \alpha f(x, y)-\alpha(\alpha-1) f(x+1, y)+\ldots \frac{(-1)^{n+1} \Gamma(\alpha+1)}{n ! \Gamma(\alpha-n+1)} f(x-1+n, y)-f(x-1, y+1) \\
&+\alpha f(x, y+1)-\frac{(\alpha)(\alpha-1)}{2} f(x+1, y+1)+\ldots \frac{(-1)^{n+1} \Gamma(\alpha+1)}{n ! \Gamma(\alpha-n+1)} f(x-1+n, y+1), \\
& G_{y}=-f(x-1, y-1)+\alpha f(x-1, y)-\frac{(\alpha)(\alpha-1)}{2} f(x-1, y+1)+\ldots \frac{(-1)^{n+1} \Gamma(\alpha+1)}{n ! \Gamma(\alpha-n+1)} f(x-1, y-1+n)- \\
& 2 f(x, y-1)+2 \alpha f(x, y)-\alpha(\alpha-1) f(x, y+1)+\ldots \frac{(-1)^{n+1} \Gamma(\alpha+1)}{n ! \Gamma(\alpha-n+1)} f(x, y-1+n)-f(x+1, y-1)+ \\
& \alpha f(x+1, y)-\frac{(\alpha)(\alpha-1)}{2} f(x+1, y+1)+\ldots \frac{(-1)^{n+1} \Gamma(\alpha+1)}{n ! \Gamma(\alpha-n+1)} f(x+1, y-1+n)-f(x-1, y)+\alpha f(x-1, y \\
&+1)+\ldots \frac{(-1)^{n+1} \Gamma(\alpha+1)}{n ! \Gamma(\alpha-n+1)} f(x-1, y+n)-2 f(x, y)+2 \alpha f(x, y+1)+\ldots \frac{(-1)^{n+1} \Gamma(\alpha+1)}{n ! \Gamma(\alpha-n+1)} f(x, y+n)-f(x+1, y)+ \\
& \alpha f(x+1, y+1)+\ldots \frac{(-1)^{n+1} \Gamma(\alpha+1)}{n ! \Gamma(\alpha-n+1)} f(x, y+n) .
\end{aligned}
$$

It is clear from Equation (8) that the GL operator depends on the signal history. By applying this operator, the resultant LS-FOM and RS-FOM filters have an infinite number of terms exhibiting the memory effect feature of the fractional-order domain as derived above. Out of these generalized equations of both filters, only $3 \times 3$ masks are displayed in Table 2, showing the proposed masks in the $x$ - and $y$-directions. The next section presents the simulation results upon employing both masks as fractional-order edge detection masks for both noise-free and noisy images to prove their efficiency in edge detection and noise robustness, highlighting their superiority over the integer-order counterparts.

\section{Simulation Results}

In this section, simulation results are being detailed for noise-free as well as noisy images upon applying both integer-order and fractional-order masks, including the proposed LS-FOM and RS-FOM for edge detection as a proof of concept.

\subsection{Noise-Free Images}

The integer-order and the fractional-order masks are applied to a set of images obtained from "The Berkley Segmentation Dataset and Benchmark" dataset [42]. The integer order filters used are the first-order ones, Sobel, Roberts, and Prewitt, and the second-order Laplacian operator. The fractional-order masks used in this part are presented in [15], FM1, FM2, and FM3 as displayed in Table 2, each mask with a specified value for the fractionalorder parameter. Moreover, the proposed masks in this work, LS-FOM and RS-FOM, are also applied to the same set of images for visual comparison. The edge detected images after simulation are shown in Figure 2. It is clear that the fractional-order filters have a higher capability in detecting more edges/details than the integer-order ones. 


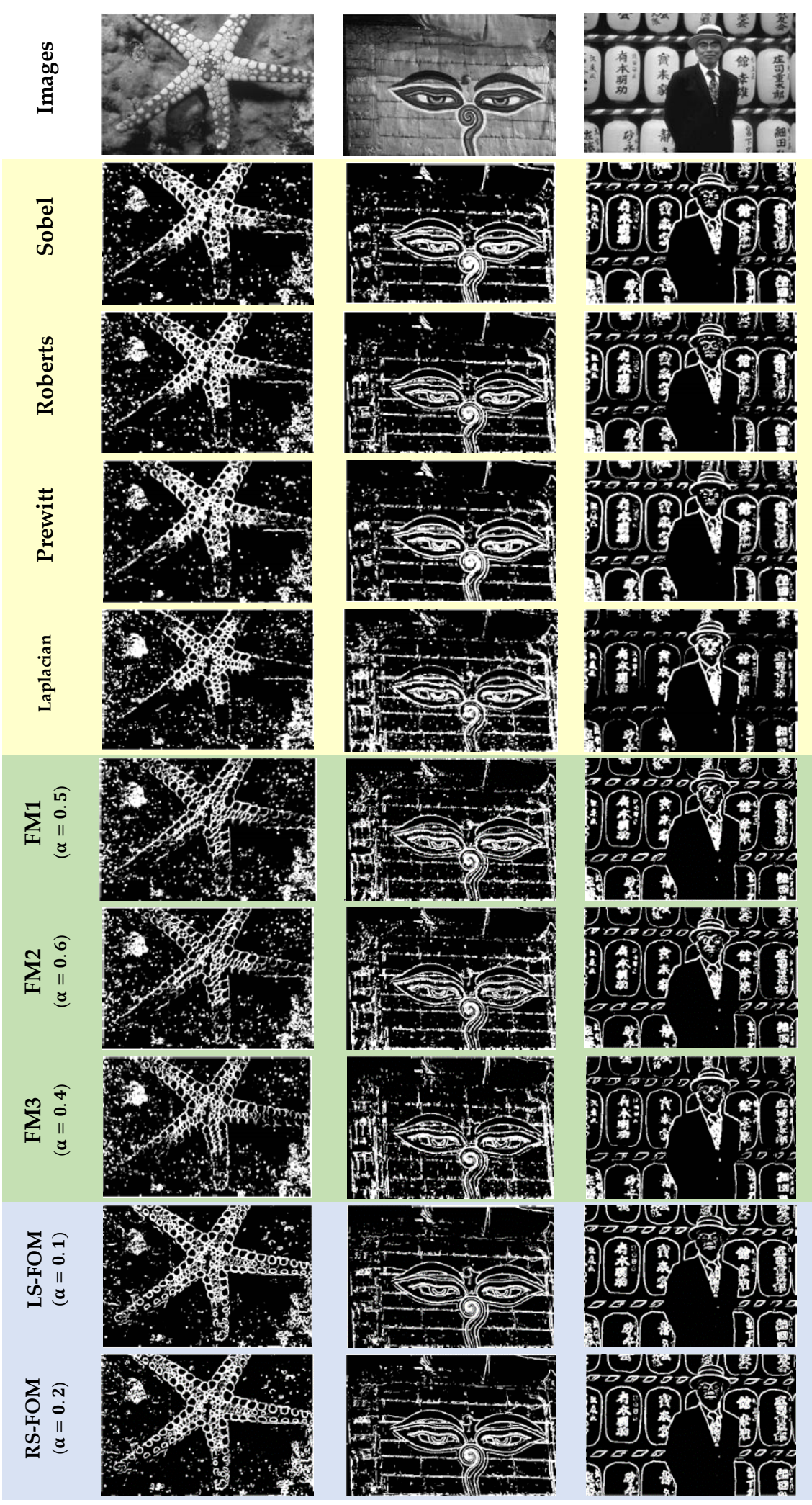

Figure 2. Integer-order versus fractional-order masks for noise-free images edge detection.

Figures 3 and 4 show the edge detected image using the LS-FOM and RS-FOM masks only, respectively, while assigning different values of the fractional-order parameter $\alpha$. Changing the value of $\alpha$, other edge maps are obtained, reaching $\alpha=1$ refers to the conventional, well-known integer order Sobel mask. It can be observed visually from 
Figure 2, that fractional-order masks can detect more details rather than their constant bitmap images obtained using their integer counterpart. To have a quantitative metric that could be proportional to the quantity of detailed detected edges, the mean intensity is calculated. Figure 5 shows the mean intensity values of the edge maps using the integerorder filters (Sobel, Prewitt and Roberts). Without a doubt, they have only one value of mean intensity, represented as the first three bars on the left hand side of the figure. The mean intensity of the same image versus $\alpha$ is also presented in Figure 5, for the three fractional-order masks (FM1, FM2, and FM3) [15], upon sweeping the value of $\alpha$ from 0.1 to 1. The figure shows that using these masks provides various detected details in the image upon tuning the required $\alpha$ set by the user, giving the designer the flexibility to "choose", or what is called an extra degree of freedom in design, which is the great advantage of fractional order operators, generally.
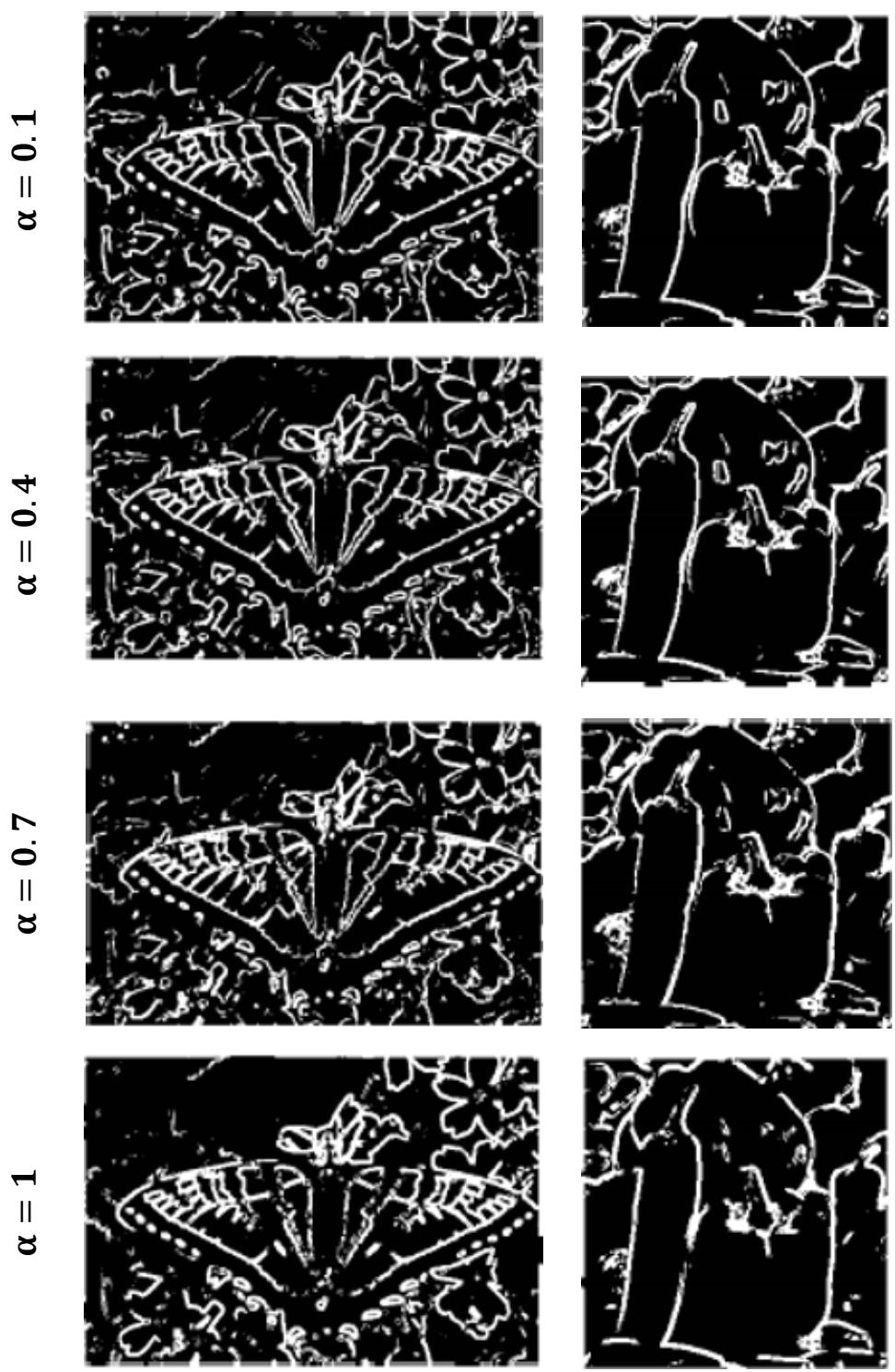

Figure 3. Edge detected images using left-sided Fractional order Sobel Mask. 

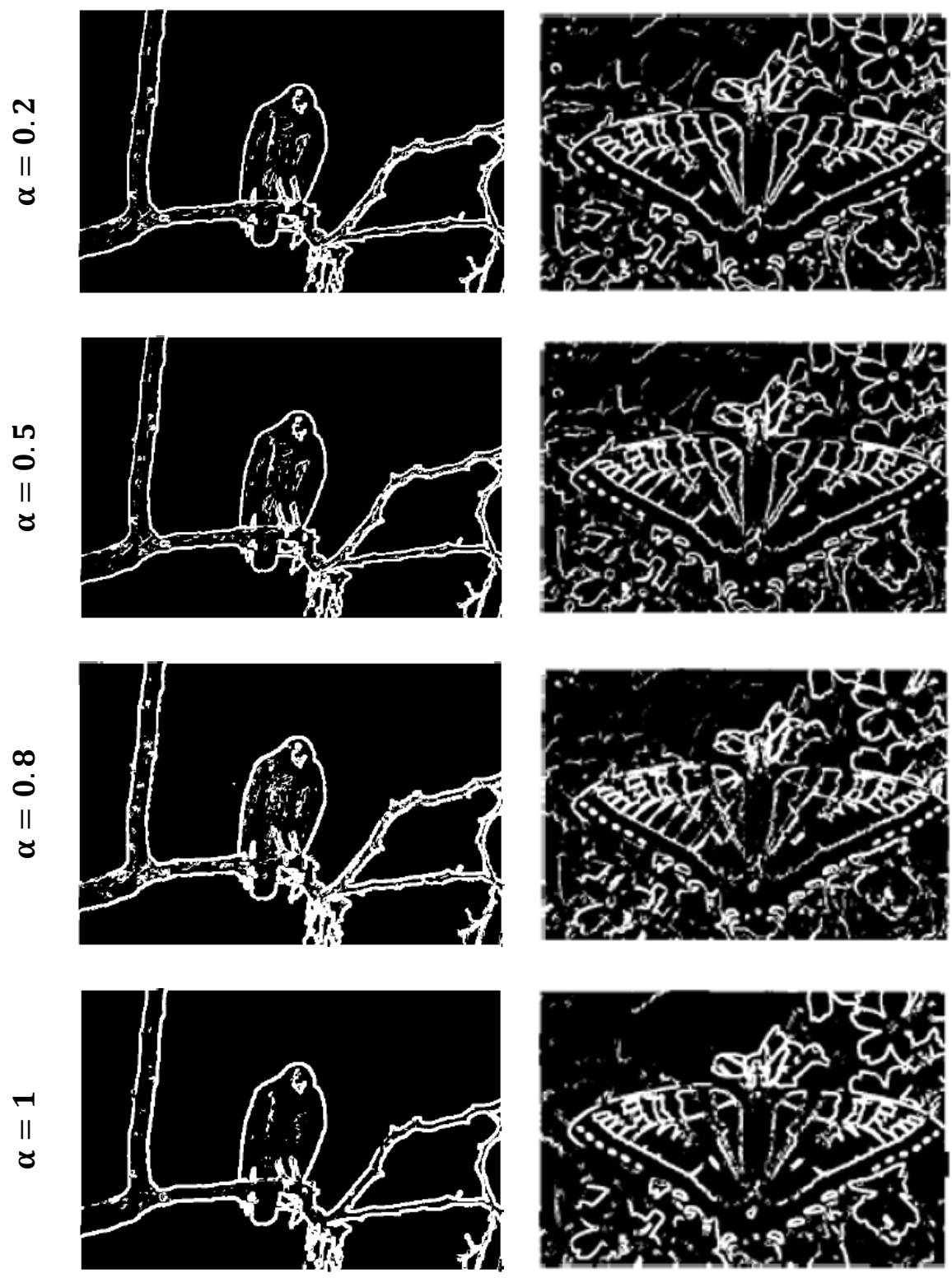

Figure 4. Edge detected images using right-sided Fractional order Sobel Mask.

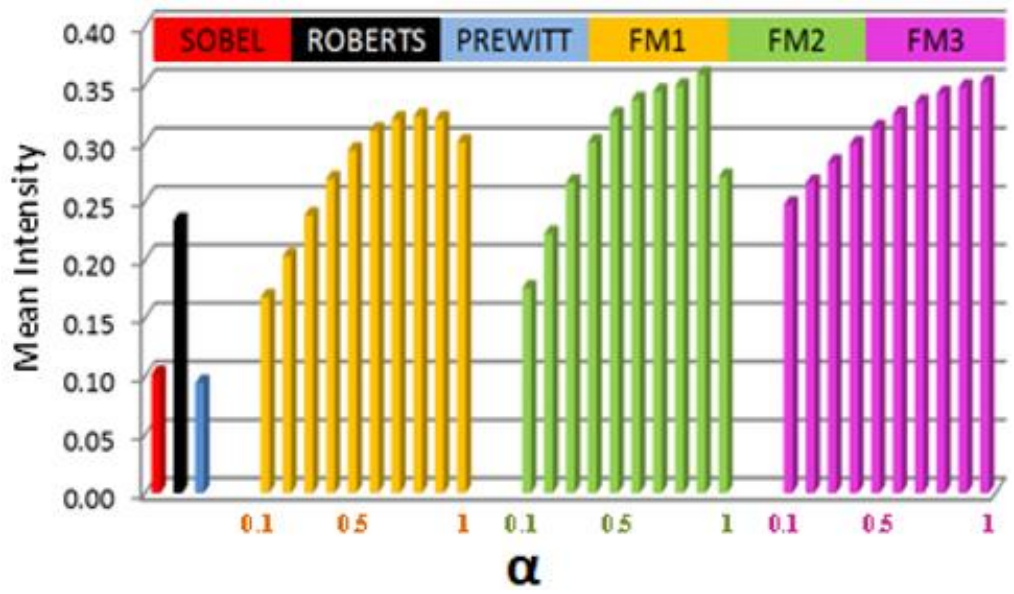

Figure 5. Mean intensity value for integer-order and fractional-order filters versus $\alpha$. 
However, one point to note here is that not all fractional-order masks have a continuous manner, meaning that on setting $\alpha=1$, it is not always guaranteed to mimic the conventional integer-order behaviour, which is apparent in Figure 5. For the three masks in comparison, none of them has the same value of mean intensity when $\alpha=1$, like any of the conventional integer order masks. The mean intensity is also calculated for the proposed RS-FOM and LS-FOM, versus their conventional Sobel mask, elaborating that for unity fractional order parameter, the same value of mean intensity is obtained, based on the continuity feature offered by the fractional-order masks proposed in this work as shown in Figure 6.

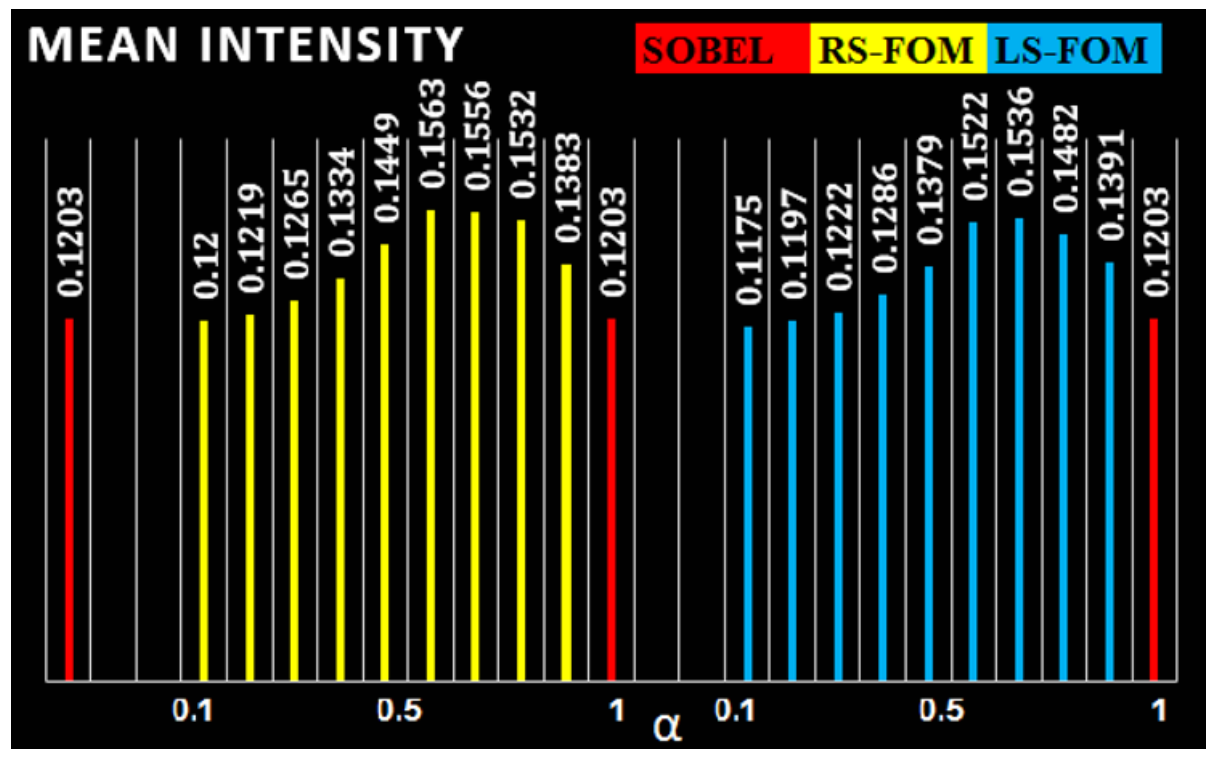

Figure 6. Mean intensity value for proposed fractional-order masks versus $\alpha$.

\subsection{Noisy Images}

In this subsection, two types of noise are added to the original images to compare different masks' capability in obtaining the edge detected images out of the noisy versions of the original ones. The Salt and Pepper (S\&P) noise is being inspected with two different densities $\mathrm{d}=0.05$ and $\mathrm{d}=0.1$, where $\mathrm{d}=0.05$ is the default value as declared by the Matlab Software. The other type is the Additive White Gaussian noise (AWGN) distribution, of zero mean with variance $\sigma=0.01$ and $\sigma=0.05$, where $\sigma=0.01$ is the default value in the Matlab Software.

It is well known that visual inspection is the primary method of comparison in the image processing field. However, in addition to that, two quantitative performance metrics are evaluated here to justify the results obtained by observation, which are the Root Mean Square Error (RMSE) and the Peak Signal to Noise Ratio (PSNR). The RMSE measures the square root of the averaged squared difference between an image and another version of this image. Generally, it is a measure of image quality. The PSNR is the term for the ratio of the maximum possible power of a signal to the corrupting noise's power affecting the presented image. In this case, the two images are the noisy edge detected image, and the edge detected one of the noiseless original images. The equations expressing these metrics are as follows:

$$
\text { RMSE }=\sqrt{\frac{1}{M N} \sum_{m=1}^{M} \sum_{n=1}^{N}\left(E \_N(n, m)-E(n, m)\right)^{2}}, \text { PSNR }=20 \log \frac{1}{\text { RMSE }^{\prime}}
$$

where $\mathrm{E} \_\mathrm{N}$ is the edge detected image out of the noisy image and $\mathrm{E}$ is the edge detected image of the noise-free original image. $\mathrm{M}$ and $\mathrm{N}$ are the image dimensions. Figure 7 summarizes the steps for noise calculation in a flowchart, where noise is added to the 
original image, choosing between the two types of noise. The masks are applied on both the noise-free original image and the noisy image, where the mask could be integer- or fractional-order. The RMSE and PSNR metrics are calculated using Equation (14) between the noise-free and the noisy edge maps obtained. "The Berkley Segmentation Dataset and Benchmark" dataset is also used in this subsection [42].

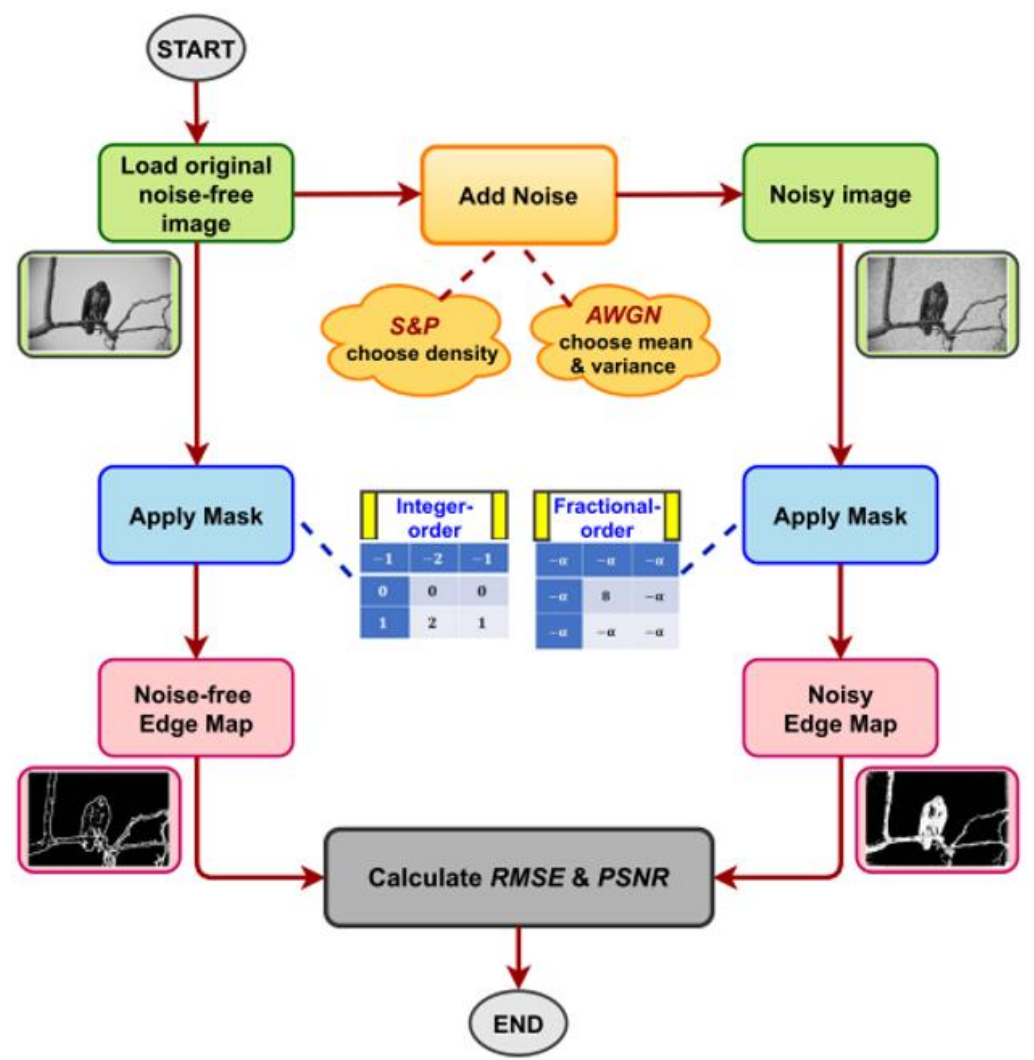

Figure 7. Noise Calculation Flowchart.

\subsubsection{Salt and Pepper Noise}

In Figure 8, the S\&P noise with density $\mathrm{d}=0.05$ is added to a set of two images (Tree and Players), and the noise performance of the integer-order filters is tested versus the fractional-order ones, including the two newly proposed masks in this work. It can be seen visually that the integer-order masks cannot have a clear edge detection out of the noisy images, unlike the fractional-order ones. For the sake of assuring the observed results quantitatively, Table 3 presents the RMSE and the PSNR values corresponding to each simulation result in Figure 8, where the numbers prove the efficiency of the fractional-order masks with less RMSE and higher PSNR than the integer-order ones.

Figure 9a shows the noisy Fly image while adding S\&P with density $d=0.05$, and the employed mask, in this case, is the right-sided fractional-order Sobel one. The edges of the noisy image are better detected for less values of fractional-order parameter $\alpha$ values than reaching the integer-order case at $\alpha=1$. Figure $9 b$ presents the noisy Bird image while adding S\&P with density $\mathrm{d}=0.1$, which is generally higher than the default value. The employed mask, in this case, is the left-sided fractional-order Sobel. The edges of the noisy image are detected more precisely for less values of the fractional-order parameter $\alpha$ values than reaching the integer-order case at $\alpha=1$. The employed threshold values are detailed in the figures as well, where they are chosen in every case to obtain the best edge detected image. 
Noisy Tree

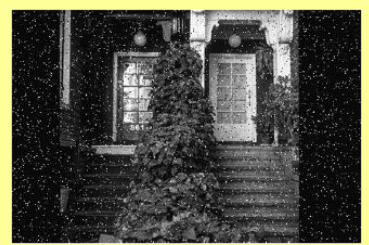

(a)

FM $1(\alpha=0.5)$

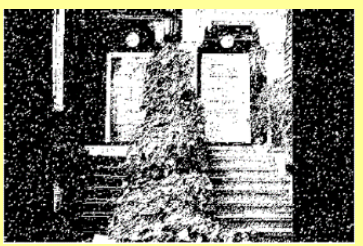

(e)

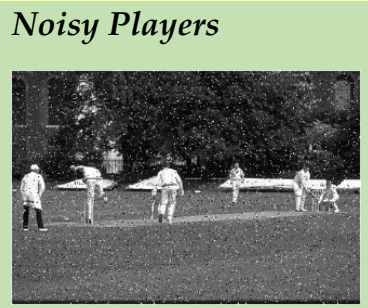

(i)

FM $(\boldsymbol{\alpha}=\mathbf{0 . 3})$

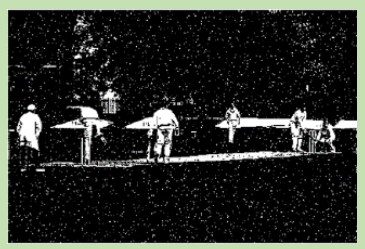

(m)
Sobel

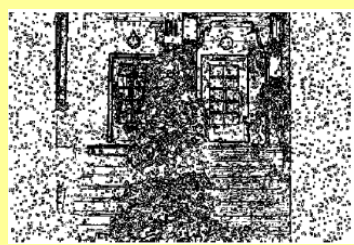

(b)

FM $2(\alpha=0.4)$

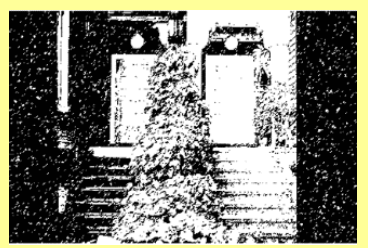

(f)

Sobel

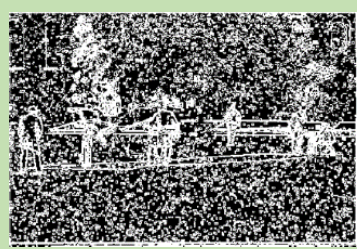

(j)

FM $(\boldsymbol{\alpha}=\mathbf{0 . 2})$

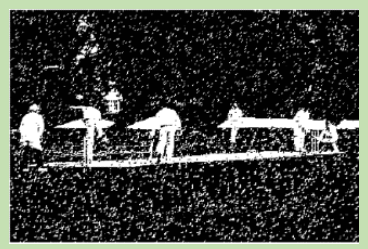

(n)
Prewitt

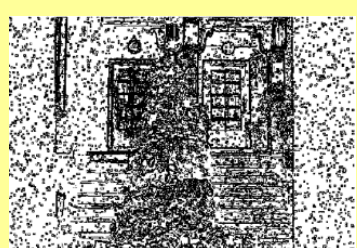

(c)

LS-FOM $(\boldsymbol{\alpha}=\mathbf{0 . 2})$

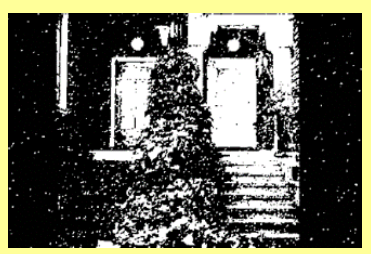

(g)

Prewitt

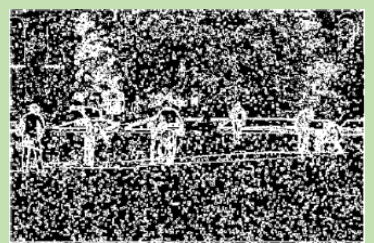

(k)

LS-FOM $(\boldsymbol{\alpha}=\mathbf{0 . 3})$

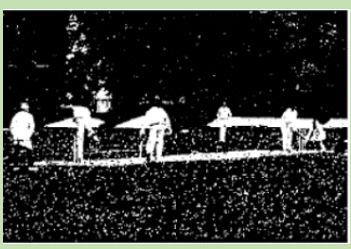

(o)
Roberts

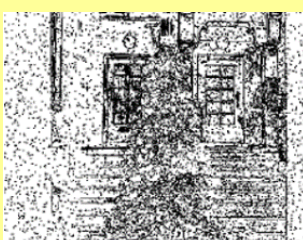

(d)

RS-FOM $(\boldsymbol{\alpha}=\mathbf{0 . 1})$

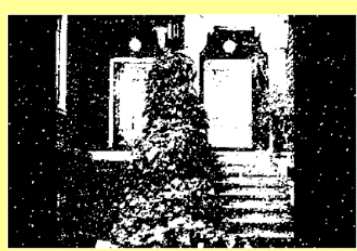

(h)

Laplacian

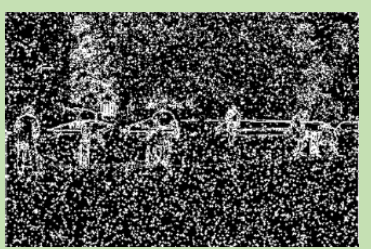

(1)

RS-FOM $(\alpha=0.2)$

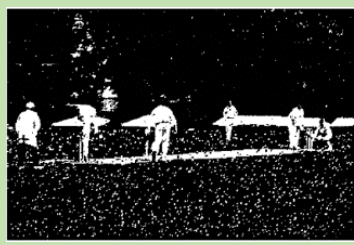

(p)

Figure 8. Edge maps of noisy images with S\&P noise added, $d=0.05,(\mathbf{a}, \mathbf{i})$ original noisy images, $(\mathbf{b}-\mathbf{d}, \mathbf{j}-\mathbf{l})$ using integer-order masks,(e) FM 1 [15], (f) FM 2 [15], (m) FM [17], (n) FM [29], (g,h,o,p) Proposed masks.

Table 3. Noise performance metrics for the Salt and Pepper (S\&P) noise added, $d=0.05$.

\begin{tabular}{|c|c|c|c|c|c|c|c|}
\hline \multicolumn{8}{|c|}{ Noisy Tree } \\
\hline \multirow[b]{2}{*}{ Masks } & \multicolumn{3}{|c|}{ Integer-order } & \multicolumn{4}{|c|}{ Fractional-order } \\
\hline & Sobel & Prewitt & Roberts & $\begin{array}{l}\text { FM1 [15] } \\
(\alpha=0.5)\end{array}$ & $\begin{array}{c}\text { FM2 [15] } \\
(\alpha=0.4)\end{array}$ & $\begin{array}{c}\text { LS-FOM } \\
(\alpha=0.2)\end{array}$ & $\begin{array}{c}\text { RS-FOM } \\
(\alpha=0.1)\end{array}$ \\
\hline \multirow{2}{*}{$\begin{array}{c}\text { RMSE } \\
\text { PSNR } \\
(\mathrm{dB})\end{array}$} & 0.4147 & 0.4135 & 0.3649 & 0.3395 & 0.3071 & 0.2219 & 0.2124 \\
\hline & 7.6459 & 7.6713 & 8.7575 & 9.3838 & 10.2534 & 13.0778 & 13.4567 \\
\hline \multicolumn{8}{|c|}{ Noisy Players } \\
\hline \multirow[b]{2}{*}{ Masks } & \multicolumn{3}{|c|}{ Integer-order } & \multicolumn{4}{|c|}{ Fractional-order } \\
\hline & Sobel & Prewitt & Laplacian & $\begin{array}{l}\text { FM [17] } \\
(\alpha=0.3)\end{array}$ & $\begin{array}{l}\text { FM [29] } \\
(\alpha=0.2)\end{array}$ & $\begin{array}{c}\text { LS-FOM } \\
(\alpha=0.3)\end{array}$ & $\begin{array}{c}\text { RS-FOM } \\
(\alpha=0.2)\end{array}$ \\
\hline RMSE & 0.4780 & 0.4744 & 0.4889 & 0.2142 & 0.3559 & 0.2207 & 0.1894 \\
\hline $\begin{array}{c}\text { PSNR } \\
(\mathrm{dB})\end{array}$ & 6.4115 & 6.4777 & 6.2164 & 13.3849 & 8.9744 & 13.1255 & 14.4522 \\
\hline
\end{tabular}


Noisy Fly

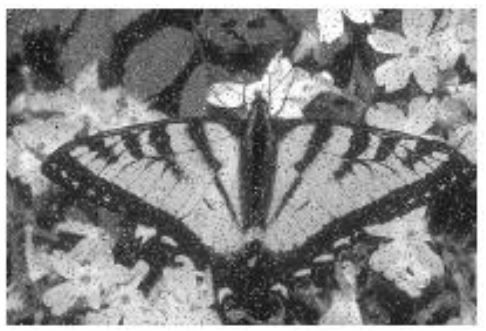

$\alpha=0.7$

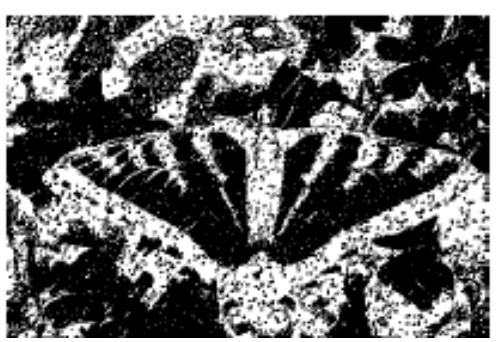

Noisy Bird

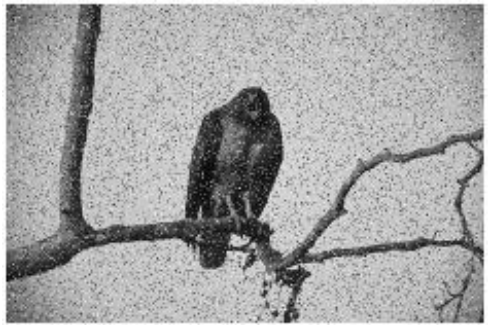

$\alpha=0.5$

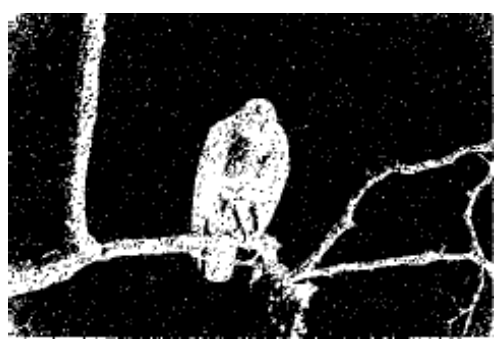

$\alpha=0.2$

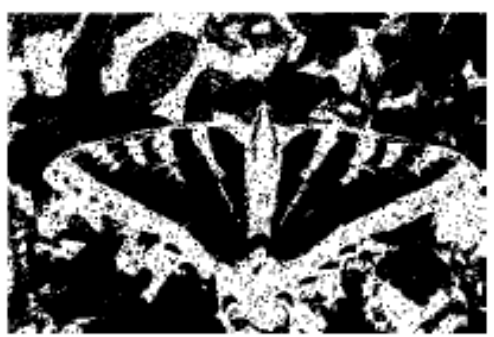

$\alpha=0.85$

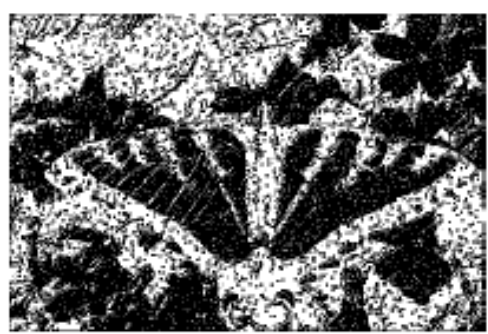

(a)

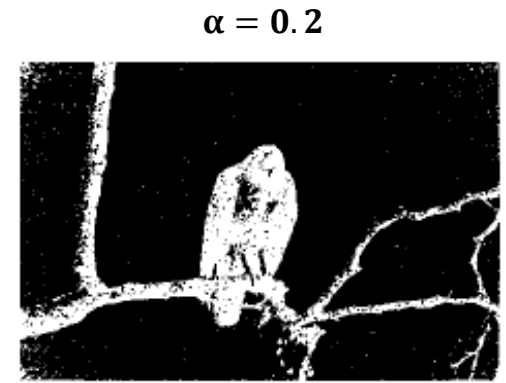

$\alpha=0.8$

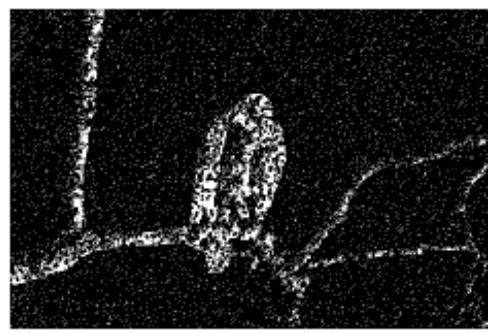

(b) $\alpha=0.5$

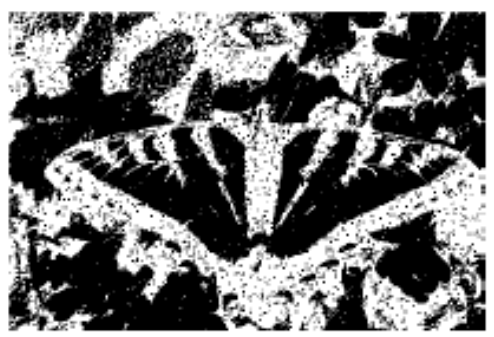

$\alpha=1$

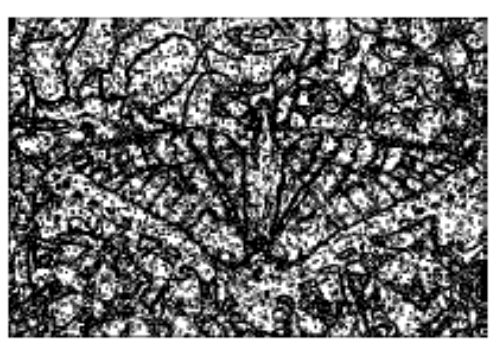

$\alpha=0.3$

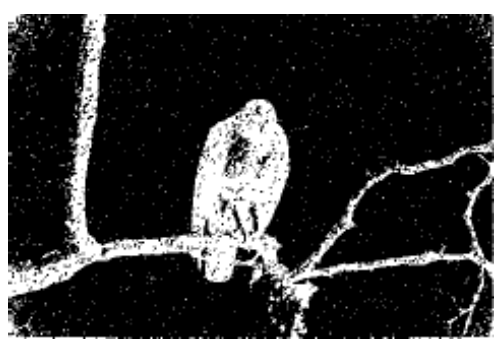

$\alpha=1$

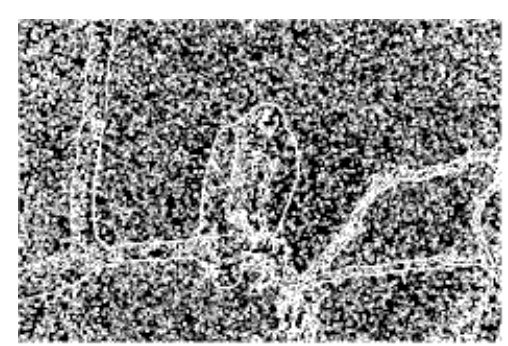

Figure 9. Edge detected images of a noisy image with S\&P noise added, (a) $d=0.1$, RS-FOM, (b) $d=0.05$, LS-FOM.

For a quantitative comparison, Figure 10 shows the numerical representation of both RMSE and PSNR for the RS-FOM and LS-FOM when applied to the Fly image after adding S\&P noise with density $d=0.1$. The results shown in Figure 10 are also for applying S\&P noise with density $d=0.05$ to the Bird image as well, proving that these results are irrespective of which images are used. The graphs show that as the fractional order parameter increases from 0.1 to 1 , the error represented by the RMSE metric increases and the PSNR decreases till reaching the worst value of each metric at $\alpha=1$, which is the integer-order case. These numbers prove the superiority of the fractional-order masks over the integer-order one regarding the noise immunity to the S\&P type of noise. 

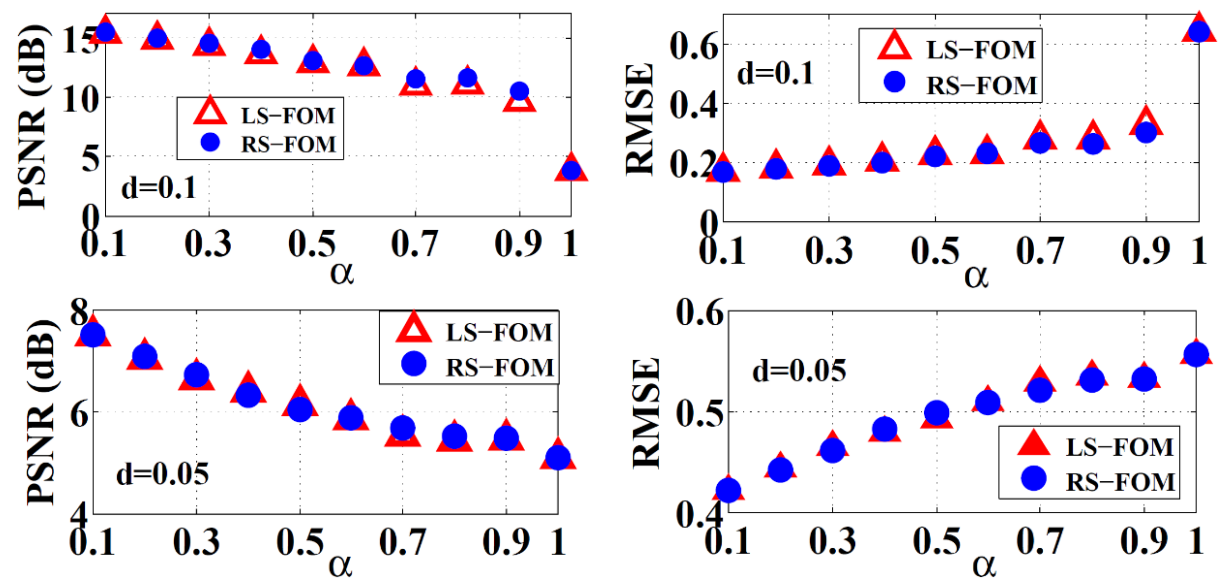

Figure 10. PSNR and RMSE for LS-FOM and RS-FOM for different densities of S\&P noisy images.

\subsubsection{Additive White Gaussian Noise}

In a similar procedure to the previous subsection, the noise performance regarding the AWGN noise is analyzed in this part. In Figure 11, the Gaussian noise is added to a set of two images (Starfish and Tower) with zero mean value and variance $\sigma=0$, and the noise performance of the integer-order filters is tested versus the fractional-order ones including the two proposed masks. The same visual observations are depicted here also, that the integer order masks cannot obtain clear versions of the edge maps out of the noisy images under test, unlike the fractional-order ones. Table 4 is presented to validate these observations numerically using the RMSE and the PSNR metrics corresponding to each simulation result in Figure 11. The numbers also prove the fractional-order masks' superiority with less RMSE and higher PSNR than the integer-order ones.

Figure 12a shows the noisy Bird image after adding Gaussian noise with zero mean value and variance $\sigma=0.01$, applying the RS-FOM for edge detection, while Figure $12 \mathrm{~b}$ includes the edge detected versions of the Pepper image applying RS-FOM, for AWGN of zero mean and variance $\sigma=0.05$. The edges of the noisy image are better seen for fewer values of the fractional-order parameter $\alpha$. Then the performance degrades as reaching the integer-order case at $\alpha=1$. Figure 13 shows the numerical representation of both RMSE and PSNR for the RS-FOM and LS-FOM when applied to the Bird image and the Pepper image with the same details explained in Figure 12. The graphs show that as the fractional order parameter increases, the RMSE increases and the PSNR decreases till reaching the worst value of each at $\alpha=1$, which is the integer-order case exactly the same case for S\&P noise discussed before. This adds that the fractional-order masks also have superior noise immunity regarding the AWGN type of noise, not only the S\&P noise.

Table 4. Noise performance metrics for Gaussian noise added, $\sigma=0.01$.

\begin{tabular}{|c|c|c|c|c|c|c|c|}
\hline \multicolumn{8}{|c|}{ Noisy Starfish } \\
\hline \multirow[b]{2}{*}{ Masks } & \multicolumn{3}{|c|}{ Integer-order } & \multicolumn{4}{|c|}{ Fractional-order } \\
\hline & Sobel & Prewitt & Roberts & $\begin{array}{c}\text { FM1 [15] } \\
(\alpha=0.3)\end{array}$ & $\begin{array}{c}\text { FM2 [15] } \\
(\alpha=0.4)\end{array}$ & $\begin{array}{c}\text { LS-FOM } \\
(\alpha=0.3)\end{array}$ & $\begin{array}{c}\text { RS-FOM } \\
(\alpha=0.4)\end{array}$ \\
\hline \multirow{2}{*}{$\begin{array}{c}\text { RMSE } \\
\text { PSNR } \\
\text { (dB) }\end{array}$} & 0.3662 & 0.3497 & 0.4066 & 0.3495 & 0.3102 & 0.2679 & 0.2512 \\
\hline & 8.7264 & 9.1265 & 7.8167 & 9.1299 & 10.1672 & 11.4411 & 12.0005 \\
\hline \multicolumn{8}{|c|}{ Noisy Tower } \\
\hline \multirow[b]{2}{*}{ Masks } & \multicolumn{3}{|c|}{ Integer-order } & \multicolumn{4}{|c|}{ Fractional-order } \\
\hline & Sobel & Prewitt & Laplacian & $\begin{array}{c}\text { FM3 [15] } \\
(\alpha=0.4)\end{array}$ & $\begin{array}{l}\text { FM [29] } \\
(\alpha=0.2) \\
\end{array}$ & $\begin{array}{c}\text { LS-FOM } \\
(\alpha=0.1)\end{array}$ & $\begin{array}{c}\text { RS-FOM } \\
(\alpha=0.4)\end{array}$ \\
\hline RMSE & 0.2525 & 0.2454 & 0.4935 & 0.2163 & 0.1664 & 0.1481 & 0.1879 \\
\hline $\begin{array}{c}\text { PSNR } \\
\text { (dB) }\end{array}$ & 11.9539 & 12.2026 & 6.1344 & 13.2993 & 15.5782 & 16.5883 & 14.5193 \\
\hline
\end{tabular}




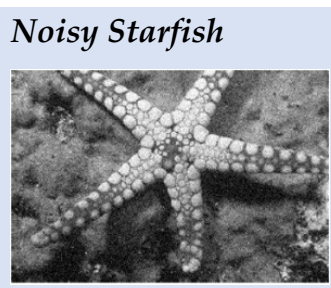

(a)

FM $1(\alpha=0.3)$

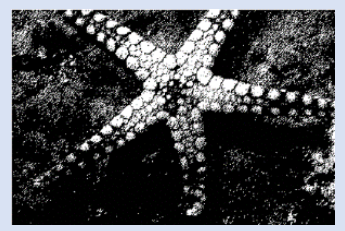

(e)

\section{Noisy Tower}

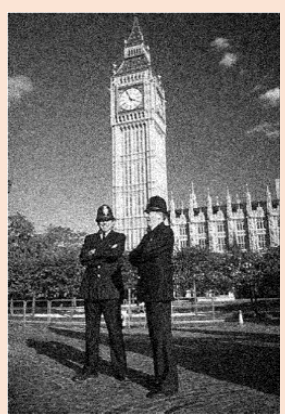

(i)

FM $3(\boldsymbol{\alpha}=\mathbf{0 . 4})$

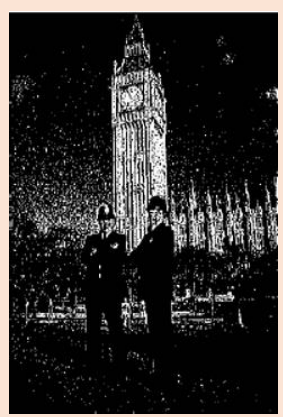

(m)

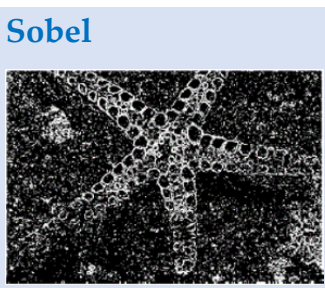

(b)

FM $2(\alpha=0.4)$

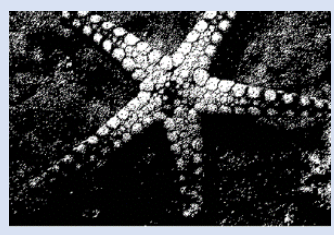

(f)

Sobel

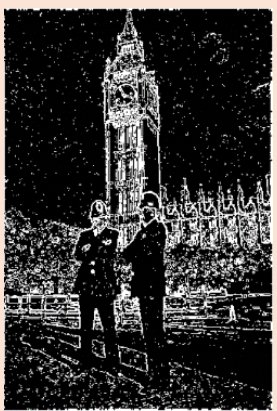

(j)

FM $(\alpha=0.2)$

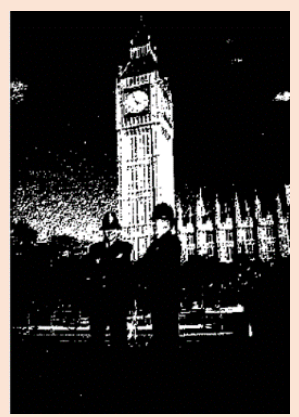

(n)

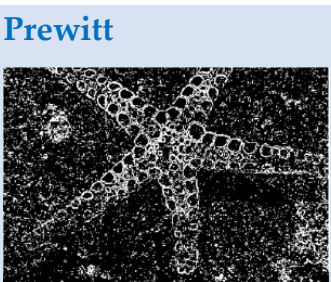

(c)

LS-FOM $(\boldsymbol{\alpha}=\mathbf{0 . 3})$

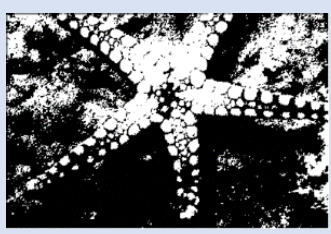

(g)

Prewitt

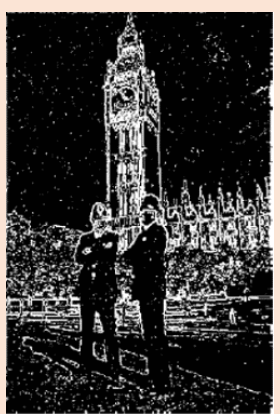

(k)

LS-FOM $(\boldsymbol{\alpha}=\mathbf{0 . 1})$

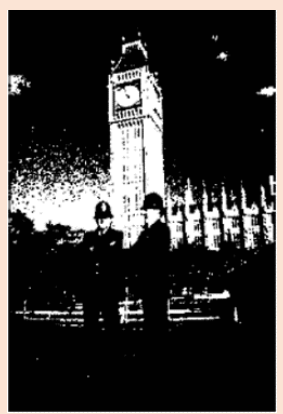

(o)

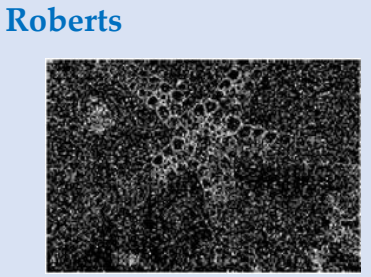

(d)

RS-FOM $(\boldsymbol{\alpha}=\mathbf{0 . 4})$

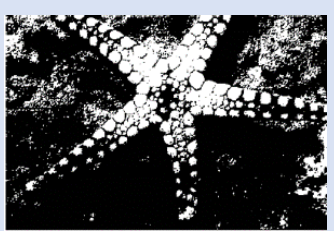

(h)

Laplacian

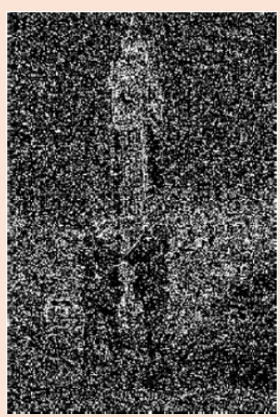

(1)

RS-FOM $(\boldsymbol{\alpha}=\mathbf{0 . 4})$

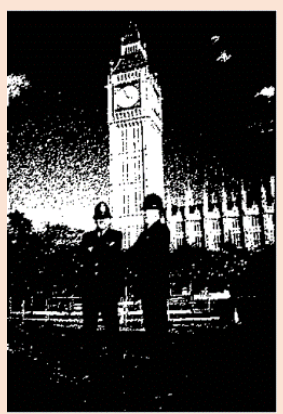

(p)

Figure 11. Edge maps of noisy images with Gaussian noise added, $\sigma=0.01$. (a,i) original noisy images, $(\mathbf{b}-\mathbf{d}, \mathbf{j}-\mathbf{1})$ integerorder masks, (e) FM 1 [15], (f) FM 2 [15], (m) FM 3 [15], (n) FM [29], (g,h,o,p) Proposed masks. 
Noisy Bird

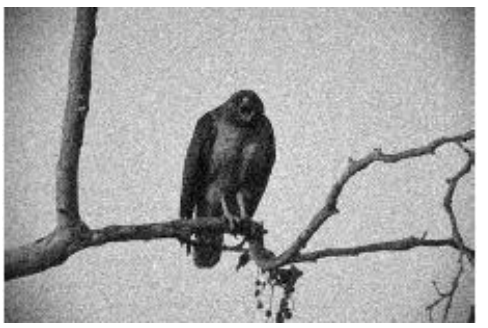

$\alpha=0.7$

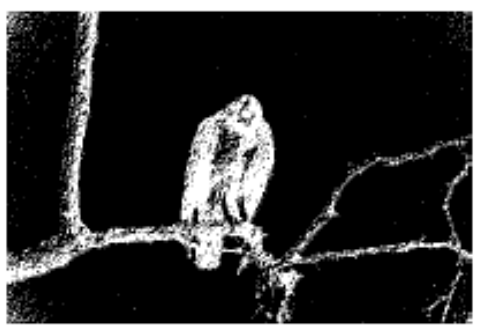

Noisy Pepper

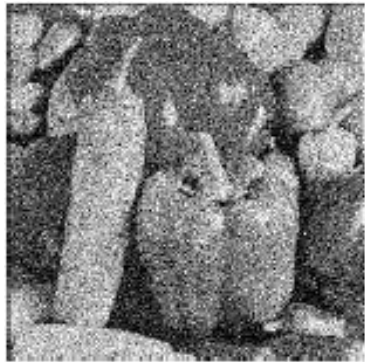

$\alpha=0.7$

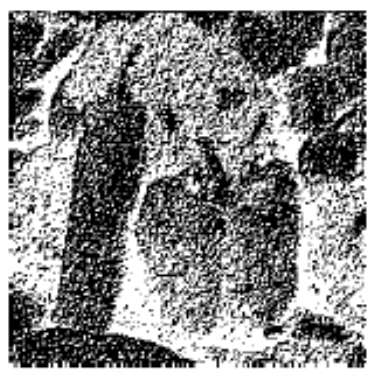

$\alpha=0.2$

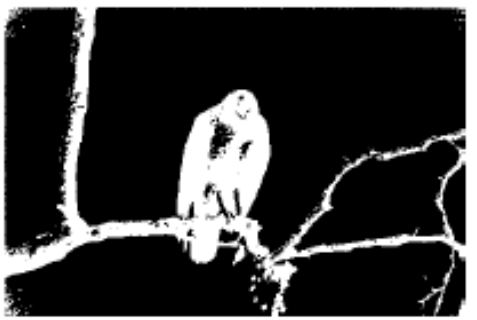

$\alpha=0.85$

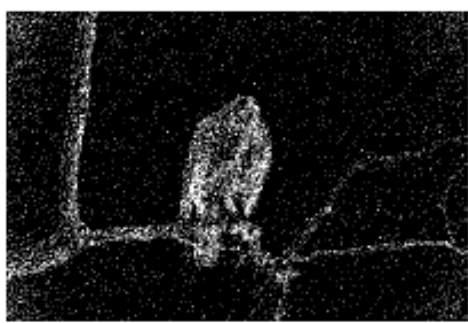

(a)

$\alpha=0.2$

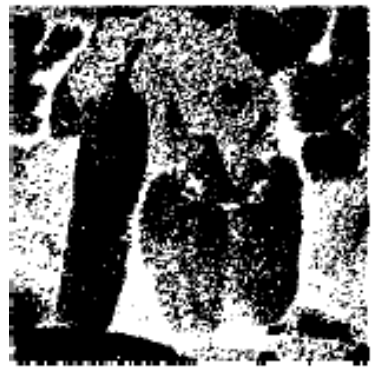

$\alpha=0.85$

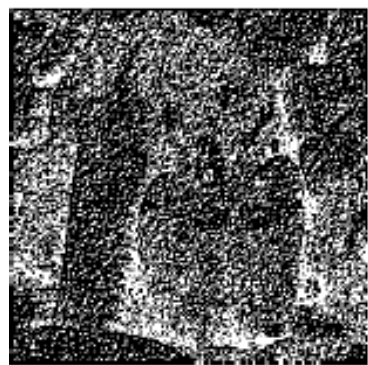

(b) $\alpha=0.5$

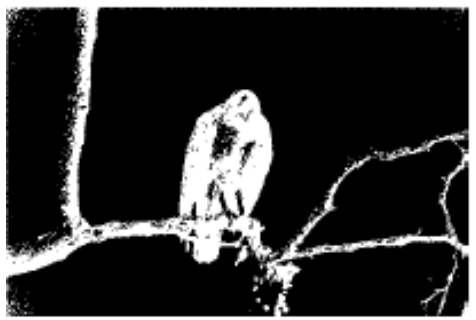

$\alpha=1$

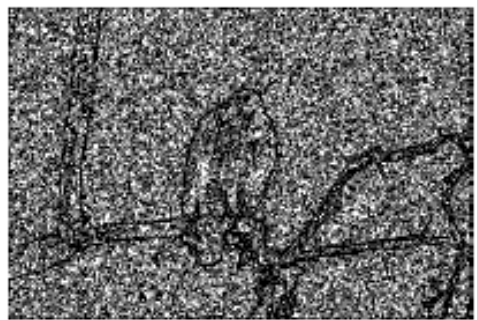

$\alpha=0.5$

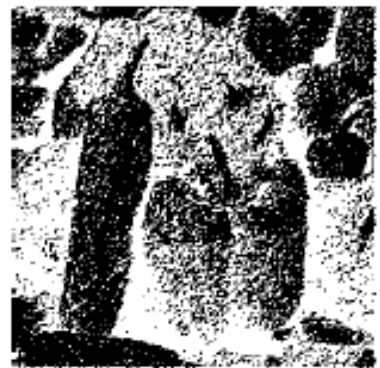

$\alpha=1$

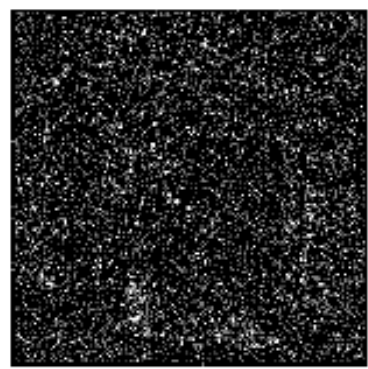

Figure 12. Edge detected images of a noisy image with Gaussian noise added, (a) $\sigma=0.01$, RS-FOM, (b) $\sigma=0.05$, LS-FOM. 

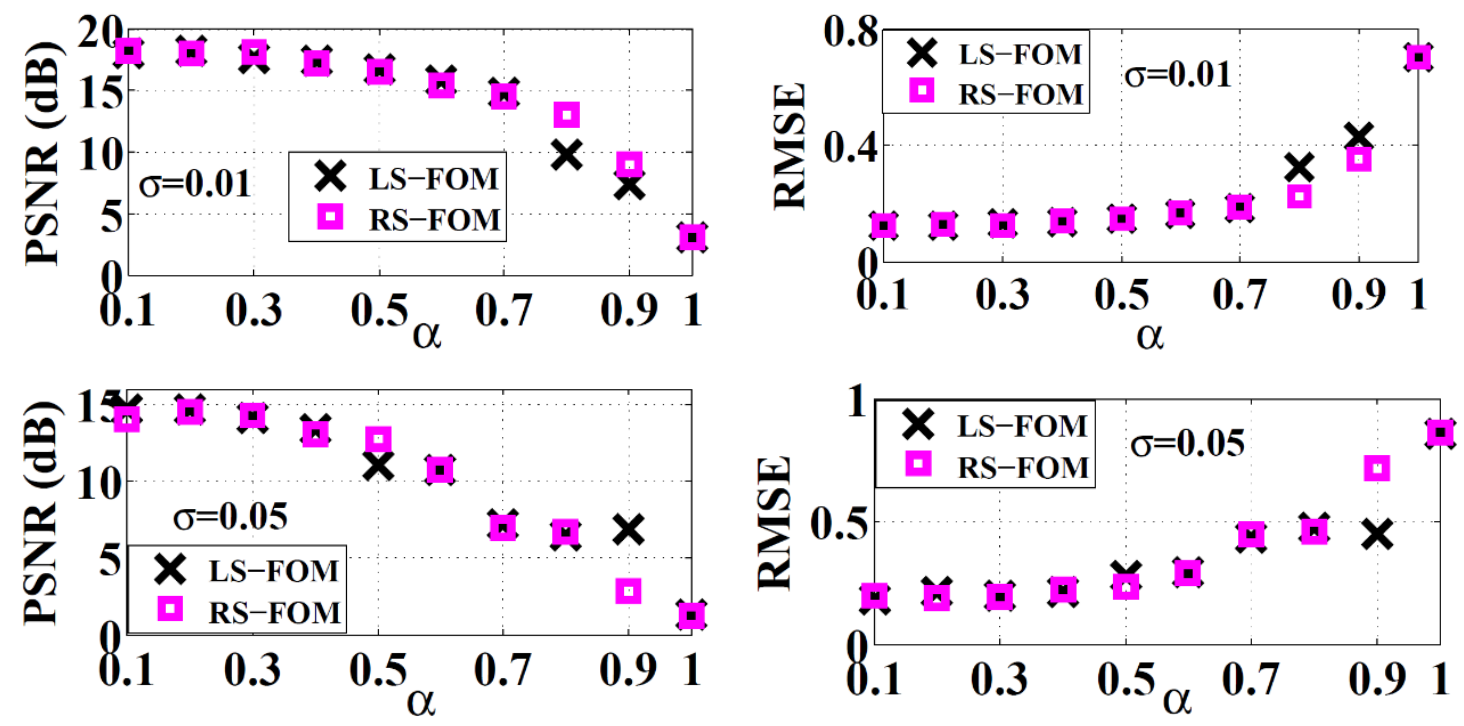

Figure 13. PSNR and RMSE for LS-FOM \& RS-FOM for different densities of Gaussian noisy images.

\section{Application: Diabetic Retinopathy Diagnosis}

As an application to the proposed fractional-order masks in the biomedical field, both masks are employed in this section to prove their efficiency in enhancing the ability to diagnose the Diabetic Retinopathy disease. Diabetes is now considered to be one of the worldwide spread chronic diseases, which may cause harmful effects to human body organs if not being well-controlled. It may cause cardiovascular diseases or may affect the nervous system or maybe the cause of some retinal abnormalities like the Diabetic Retinopathy (DR). Diagnosing the DR disease in an early stage is a crucial issue, as the severity of such disease increases with time, which may lead to vision loss or even blindness in late stages. Figure 14a shows an image of a normal retina, while minor changes in the retina's blood vessels start to appear in the early stage of DR; dilatations in the retinal capillaries called microaneurysms can be detected as shown in Figure 14b. They are considered to be the earliest visible sign of DR, and they appear as small red dots. Weak retinal blood vessels may start to leak or rupture, leading to hemorrhage, as shown in Figure 14c, which is also detected in the early stages of DR. As time passes, lipid leakage from capillaries may start to take place, resulting in hard exudates which look like yellow-white deposits of variable shapes in the retina. Soft exudates occur in the case of retinal ischemia due to reduced blood flow to the retina and, sometimes called cotton wool spots presented in Figure 14d [43]. Progressively, new fragile bleeding blood vessels start to appear, resulting in neovascularization, causing vision loss. Early detection of DR symptoms could help in the patient's treatment and thus limiting the progress of the disease.

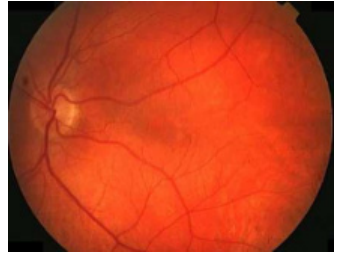

(a)

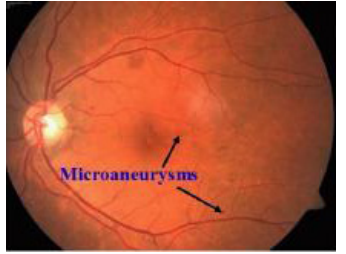

(b)

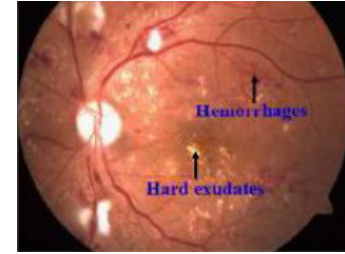

(c)

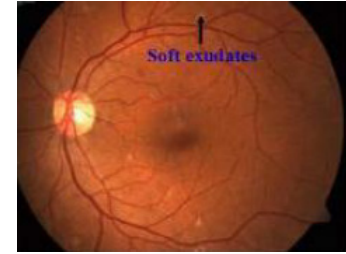

(d)

Figure 14. Diabetic Retinopathy symptoms: (a) normal retina, (b) microaneurysms, (c) hemorrhage and hard exudates and (d) soft exudates [43].

Detecting the abnormalities in medical images in details helps in the early diagnosis of DR saving patients from disease progression [44]. It is a challenging task to efficiently segment medical images, as these images may suffer from noise, poor contrasts, or diffused 
boundaries or edges. Edge detection is considered a basic step in edge-based segmentation techniques, and it would be beneficial if the noise imposed on the image is reduced in a preprocessing step-like edge detection [45]. Different methods were documented in literature for detecting DR signs, thus its diagnosis. For example, an edge sharpening method was proposed in [46] to simplify the segmentation process of hard and soft exudates detection. In [47], preprocessing steps using Canny and Kirsch templates edge detection techniques were presented to enhance the blood vessels segmentation of DR retinal images. Furthermore, a combination of edge detection and threshold techniques was introduced to eliminate noise from fundus images, which helped in exudates detection more accurately in [48]. Moreover, microaneurysms detection was proposed in [49] using Canny's edge detector as a step for image segmentation. Hence, employing fractional-order edge detection filters could greatly enhance the performance of any edge-based technique used especially for DR diagnosis based on their superior noise performance over integer ones, as explained in previous sections, showing more texture details of retinal images improving the disease progress detection.

The dataset used in this work for DR diagnosis is the online database of the STARE (STructured Analysis of the Retina) Project [50]. Figure 15 shows the edge detected images using integer order filters versus different values of $\alpha$ of the fractional mask FM2 [15] for the same DR image. It is apparent that fractional-order filters are more sensitive to edge changes than the conventional ones showing more texture details of retinal images which improve the disease progress detection. The proposed fractional-order masks are employed here for edge detection of the same retina image of a DR patient, for different values of $\alpha$ ranging from 0.1 to 1 , where unity represents the conventional Sobel mask, and the results are presented in Figure 16. The figure shows that different fractional-order parameter detects more edges showing more details that would not have been detected in the integer-order case, which enhances the diagnosis of the disease.

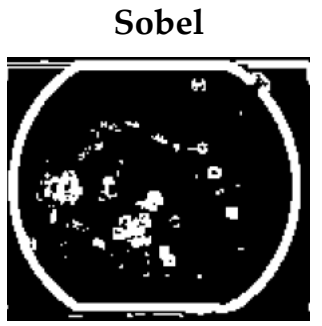

FM2

$\alpha=0.2$
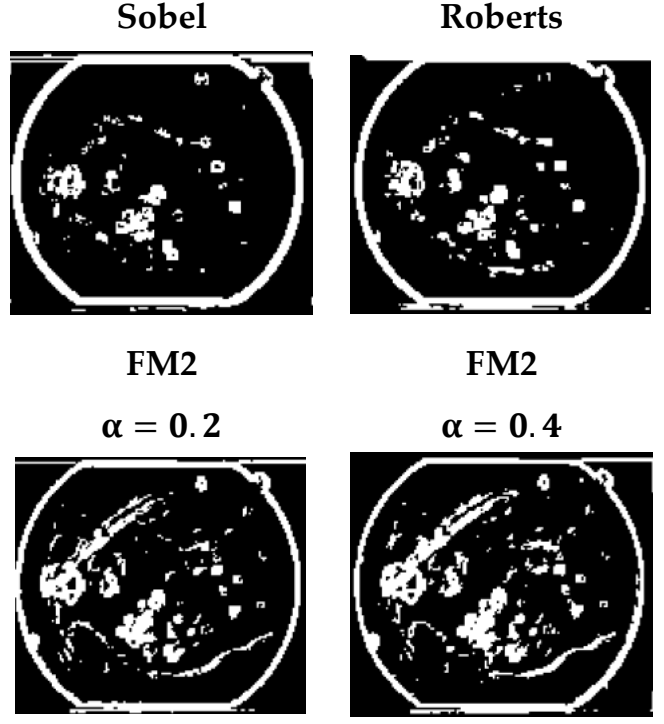

FM2

$\alpha=0.4$

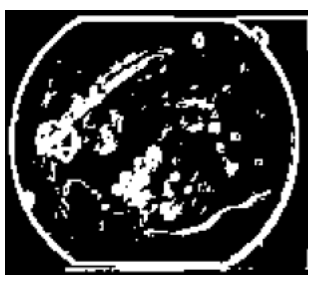

Figure 15. Integer versus Fractional-order mask.

\section{Prewitt}

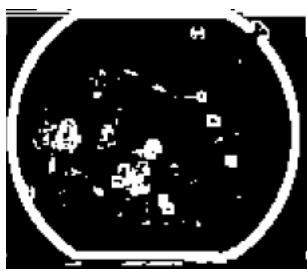

FM2

$\alpha=0.7$

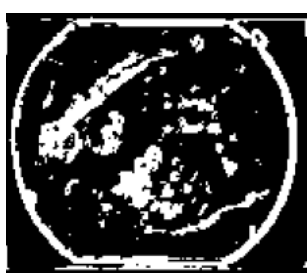


$\alpha=0.2$

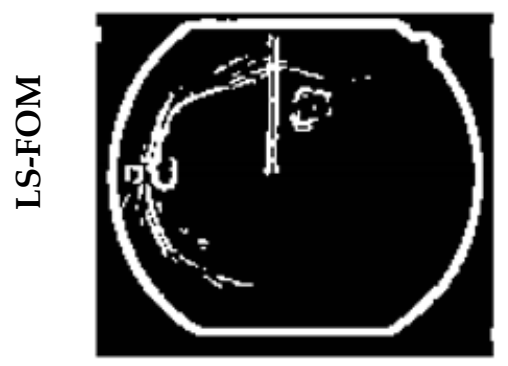

$\alpha=\mathbf{0 . 1}$

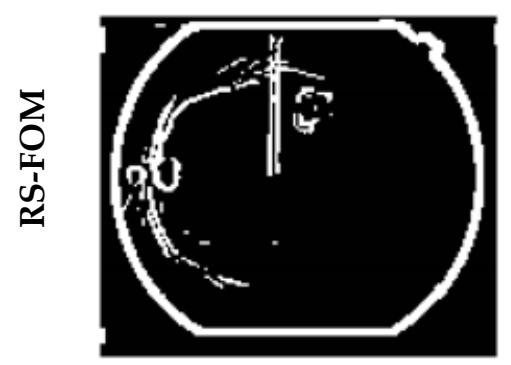

$\alpha=0.7$

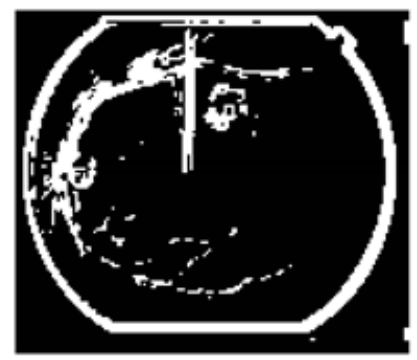

$\alpha=0.6$

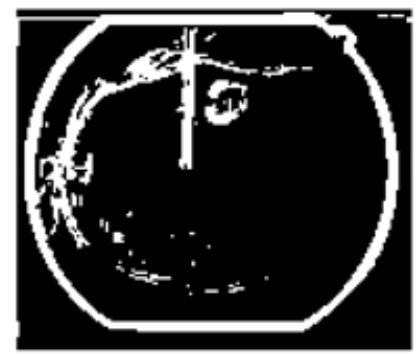

$\alpha=1$

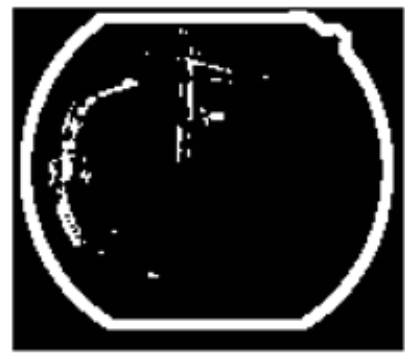

$\alpha=1$

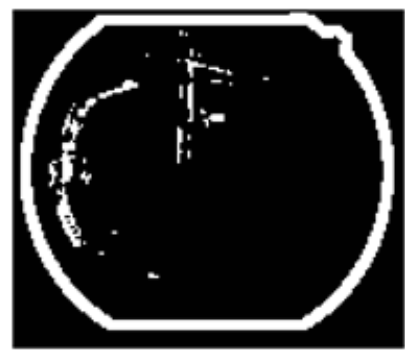

Figure 16. Proposed RS-FOM and LS-FOM versus integer Sobel mask.

Three groups of DR retinal images were chosen for testing from the STARE dataset based on the ophthalmologists' diagnosis recorded in this dataset. The selected images suffer from only DR disease, and they were divided into three groups according to the progress level of the disease. The three groups are Normal, Early Stage Background DR called BDR and Late Stage Proliferative DR called PDR; each group consists of 12 images, but only 3 images of each group are illustrated here for elaboration. Table 5 shows the results of three images of each group when subjected to edge detection using integer and fractional order masks as well as the proposed LS-FOM for $\alpha=0.4$ and RS-FOM for $\alpha=0.6$. It is clear that the integer-order masks miss many details in the images, which will lead to an incorrect diagnosis of the progress of the DR disease. The mean value of the intensity of the edge detected images is an indication of the amount of details detected as edges.

The mean intensity values are tabulated in Table 6, corresponding to each case shown in Table 5. In order to interpret the numerical results correctly, there is one thing to note here, which is the drawback of the integer-order filters of producing thick edges, which may lead to a higher value of mean intensity due to the thick edges rather than the number of useful details detected. However, the fractional-order filters do not produce thick edges; hence the value of the mean intensity directly represents the fine details detected through these filters, enhancing the DR progress follow up.

Finally, real cases of DR patients for four cases are described in Figure 17. These cases are previously diagnosed by ophthalmologists as Late-Stage PDR. The first case is for a 53-year-old male diagnosed with PDR, Diabetic macular edema, and tractional retinal detachment. The second case is for a 45-year-old patient who suffers from PDR, with small hyperfluorescent dots (microaneurysms), and blockage from a subhyaloid hemorrhage. The third case is for a patient that has an advanced PDR in his left eye, while the last case is for a 39-year-old female suffering from long standing diabetes PDR with microaneurysms and neovascularization. The images are edge detected using the LS-FOM of $\alpha=0.7$ and RS-FOM of $\alpha=0.5$. The edge detected images show obviously the details of the retina effectively for these late stages of DR. 
Table 5. Diabetic Retinopathy (DR) edge detected images using proposed fractional-order masks.

\begin{tabular}{|c|c|c|c|c|c|c|c|c|c|}
\hline & \multicolumn{3}{|c|}{ Normal } & \multicolumn{3}{|c|}{ Early Stage DR } & \multicolumn{3}{|c|}{ Late Stage DR } \\
\hline & NOR1 & NOR2 & NOR3 & BDR1 & BDR2 & BDR & PDR1 & PDR2 & PDR3 \\
\hline Color & & & & & & & 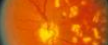 & & \\
\hline Grey & & & & 4 & & & & & \\
\hline Sobel & & & & & & & & & \\
\hline Robert & & & & & & & & & \\
\hline $\begin{array}{c}\text { FM1 } \\
\alpha=0.2\end{array}$ & & & & & & & & & \\
\hline $\begin{array}{c}\text { FM2 } \\
\alpha=0.3\end{array}$ & & & & & & & & & \\
\hline $\begin{array}{c}\text { FM3 } \\
\alpha=0.4\end{array}$ & & & 4 & & & & & & \\
\hline LS-FOM & & & & & & & & & \\
\hline $\begin{array}{c}\text { RS-FOM } \\
\alpha=0.6\end{array}$ & & 6 & & & & & & & 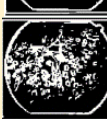 \\
\hline
\end{tabular}

Table 6. Mean Intensity values for DR edge detected images.

\begin{tabular}{cccccccccc}
\hline & \multicolumn{3}{c}{ Normal } & \multicolumn{3}{c}{ BDR } & \multicolumn{3}{c}{ PDR } \\
\hline & NOR1 & NOR2 & NOR3 & BDR1 & BDR2 & BDR3 & PDR1 & PDR2 & PDR3 \\
\hline Sobel & 0.1063 & 0.1103 & 0.1075 & 0.2014 & 0.1737 & 0.1833 & 0.1808 & 0.3097 & 0.2763 \\
\hline Robert & 0.0939 & 0.1037 & 0.0944 & 0.1782 & 0.1606 & 0.1440 & 0.1546 & 0.2717 & 0.2564 \\
\hline $\begin{array}{c}\text { FM1 } \\
\boldsymbol{\alpha}=\mathbf{0 . 2}\end{array}$ & 0.0905 & 0.0953 & 0.0917 & 0.1603 & 0.1554 & 0.1569 & 0.2270 & 0.2796 & 0.2652 \\
\hline $\begin{array}{c}\text { FM2 } \\
\boldsymbol{\alpha = \mathbf { 0 . 3 }}\end{array}$ & 0.0916 & 0.0920 & 0.0877 & 0.1637 & 0.1567 & 0.1644 & 0.2162 & 0.2841 & 0.2758 \\
\hline $\begin{array}{c}\text { FM3 } \\
\boldsymbol{\alpha}=\mathbf{0 . 4}\end{array}$ & 0.0839 & 0.0841 & 0.0775 & 0.1581 & 0.1579 & 0.1576 & 0.2096 & 0.2726 & 0.2819 \\
\hline $\begin{array}{c}\text { LS- } \\
\text { FOM } \\
\boldsymbol{\alpha}=\mathbf{0 . 4}\end{array}$ & $\mathbf{0 . 0 9 9 0}$ & $\mathbf{0 . 0 9 5 9}$ & $\mathbf{0 . 0 9 1 9}$ & $\mathbf{0 . 1 6 6 6}$ & $\mathbf{0 . 1 5 8 5}$ & $\mathbf{0 . 1 6 7 0}$ & $\mathbf{0 . 2 2 6 4}$ & $\mathbf{0 . 2 8 3 8}$ & $\mathbf{0 . 2 6 6 8}$ \\
\hline $\begin{array}{c}\text { RS- } \\
\text { FOM } \\
\boldsymbol{\alpha}=\mathbf{0 . 6}\end{array}$ & $\mathbf{0 . 1 2 3 7}$ & $\mathbf{0 . 1 2 3 4}$ & $\mathbf{0 . 1 0 8 8}$ & $\mathbf{0 . 1 5 9 1}$ & $\mathbf{0 . 1 6 1 7}$ & $\mathbf{0 . 1 6 2 6}$ & $\mathbf{0 . 2 3 0 6}$ & $\mathbf{0 . 2 6 5 9}$ & $\mathbf{0 . 2 5 7 1}$ \\
\hline
\end{tabular}



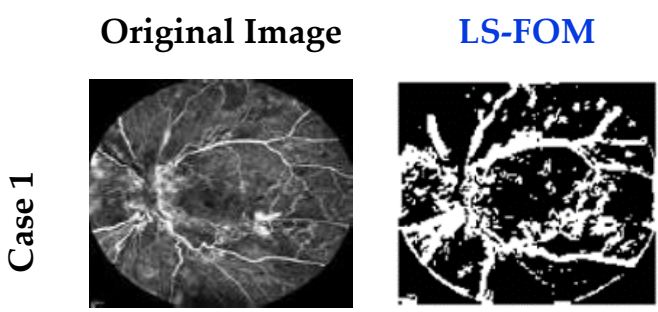

\section{RS-FOM}
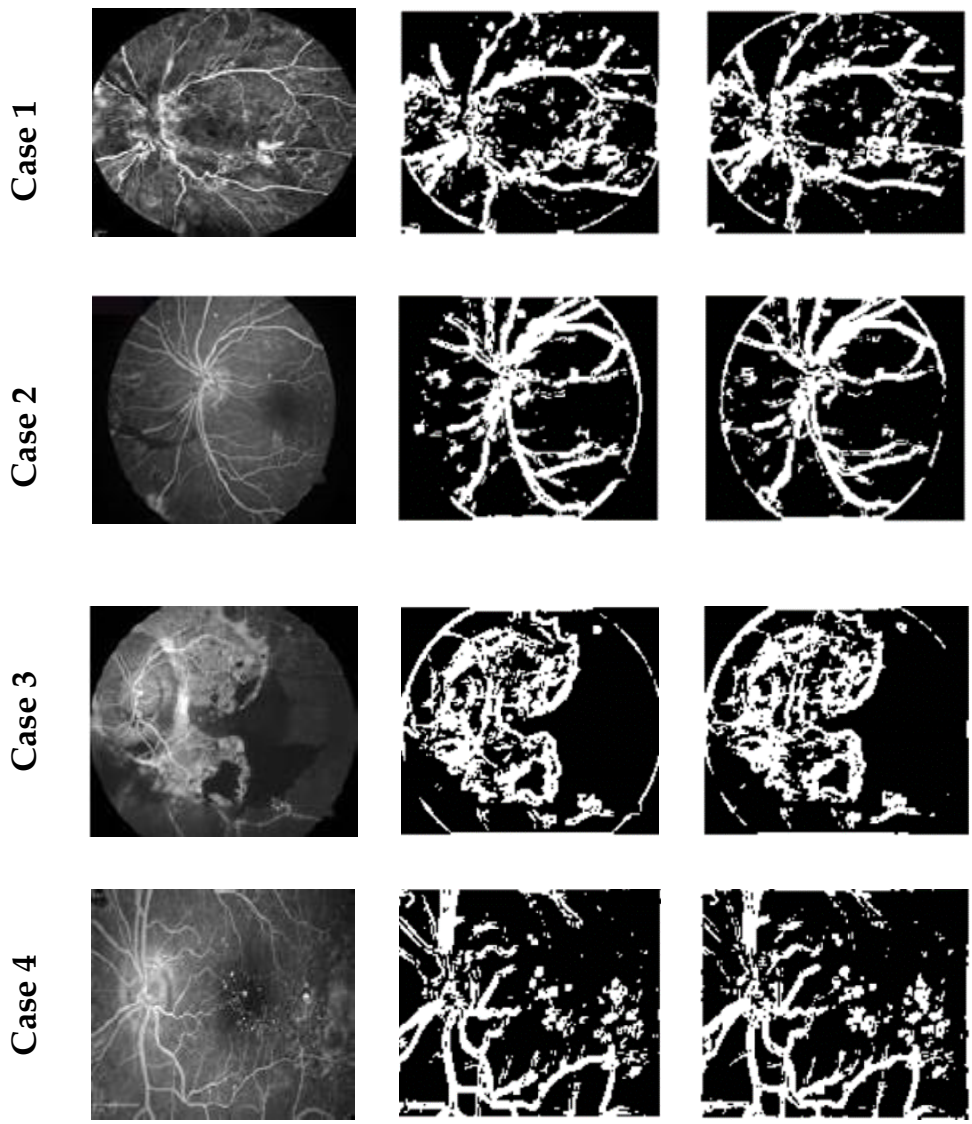

Figure 17. Practical DR real case studies.

\section{Conclusions}

Two novel fractional-order masks are proposed in this work with their mathematical proofs based on the Grunwald-Letnikov fractional-order derivative. The fractional-order masks are employed in image edge detection, where the fractional-order parameter offers an extra degree of freedom to detect more fine details with sharper edges. The noise performance of the two masks is evaluated with respect to Salt and Pepper noise and Gaussian noise. The fractional-order masks exhibited superior performance than the integer-order masks regarding both types of noise having higher PSNR for less fractional order parameter compared to integer-order operators. As a practical application, the fractional-order edge masks are employed in diagnosing the Diabetic Retinopathy disease using the STARE database images and followed by testing images of real patients' cases. Based on the results obtained in this work, the two proposed fractional-order masks can contribute in enhancing the noise robustness of any other image processing application, such as encryption and segmentation.

Author Contributions: Conceptualization, S.M.I., L.A.S., A.H.M. and A.G.R.; formal analysis, S.M.I., and L.A.S.; funding acquisition, A.G.R.; methodology, S.M.I., L.A.S., A.H.M. and A.G.R.; software, S.M.I.; supervision, A.H.M. and A.G.R.; validation, S.M.I.; Writing-original draft, S.M.I.; writingreview \& editing, L.A.S., A.H.M. and A.G.R. All authors have read and agreed to the published version of the manuscript.

Funding: This paper is based upon work supported by Science, Technology, and Innovation Funding Authority (STIFA) under grant (\#25977).

Institutional Review Board Statement: Not applicable.

Informed Consent Statement: Not applicable. 
Data Availability Statement: Publicly available datasets were analyzed in this study. This data can be found here: https://www2.eecs.berkeley.edu/Research/Projects/CS/vision/grouping/segbench/ (accessed on 5 March 2021) and http://www.ces.clemson.edu/-ahoover/stare (accessed on 5 March 2021).

Conflicts of Interest: The authors declare no conflict of interest.

\section{Abbreviations}

$\begin{array}{llll}\text { FC } & \text { Fractional Calculus } & \text { S\&P } & \text { Salt and Pepper } \\ \text { GL } & \text { Grunwald-Letnikov } & \text { AWGN } & \text { Additive White Gaussian noise } \\ \text { FO } & \text { Fractional Order } & \text { RMSE } & \text { Root Mean Square Error } \\ \text { LS-FOM } & \text { left-sided fractional-order mask } & \text { PSNR } & \text { Peak Signal to Noise Ratio } \\ \text { RS-FOM } & \text { right-sided fractional-order mask } & \text { DR } & \text { Diabetic Retinopathy } \\ \text { FM } & \text { Fractional Mask } & \text { BDR } & \text { Background Diabetic Retinopathy } \\ \text { MRI } & \text { Magnetic Resonance Imaging } & \text { PDR } & \text { Proliferative Diabetic Retinopathy } \\ \text { AD } & \text { Alzheimer disease } & \text { STARE } & \text { STructured Analysis of the Retina }\end{array}$

\section{References}

1. Gonzalez, R.C.; Woods, R.E. Digital Image Processing, 3rd ed.; Prentice-Hall, Inc.: Upper Saddle River, NJ, USA, 2006.

2. Torre, V.; Poggio, T.A. On edge detection. IEEE Trans. Pattern Anal. Mach. Intell. 1986, 147-163. [CrossRef]

3. Oldham, K.B.; Spanier, J. The Fractional Calculus: Theory and Applications of Differentiation and Integration to Arbitrary Order; Dover Books on Mathematics; Elsevier: Amsterdam, The Netherlands, 2006.

4. Machado, J.A.T.; Kiryakova, V. Recent history of the fractional calculus: Data and statistics. In Basic Theory; Kochubei, A., Luchko, Y., Eds.; De Gruyter: Berlin, Germany, 2019; pp. 1-22.

5. Elwy, O.; Abdelaty, A.; Said, L.; Radwan, A. Fractional calculus definitions, approximations, and engineering applications. J. Eng. Appl. Sci. 2020, 67, 1-30.

6. Allagui, A.; Freeborn, T.J.; Elwakil, A.S.; Fouda, M.E.; Maundy, B.J.; Radwan, A.G.; Said, Z.; Abdelkareem, M.A. Review of fractional-order electrical characterization of supercapacitors. J. Power Sources 2018, 400, 457-467. [CrossRef]

7. Helmy, A.H.; Ismail, S.M. Fractional-Order Image Edge Detector on FPGA. In Proceedings of the 2018 30th International Conference on Microelectronics (ICM), Sousse, Tunisia, 16-19 December 2018; pp. 339-342. [CrossRef]

8. Said, L.A.; Radwan, A.G.; Madian, A.H.; Soliman, A.M. Three fractional-order-capacitors-based oscillators with controllable phase and frequency. J. Circuits Syst. Comput. 2017, 26, 1750160. [CrossRef]

9. Tolba, M.F.; Elsafty, A.H.; Armanyos, M.; Said, L.A.; Madian, A.H.; Radwan, A.G. Synchronization and FPGA realization of fractional-order Izhikevich neuron model. Microelectron. J. 2019, 89, 56-69. [CrossRef]

10. Ismail, S.M.; Said, L.A.; Radwan, A.G.; Madian, A.H.; Abu-ElYazeed, M.F. A novel image encryption system merging fractionalorder edge detection and generalized chaotic maps. Signal Process. 2020, 167, 107280. [CrossRef]

11. Soliman, N.S.; Tolba, M.F.; Said, L.A.; Madian, A.H.; Radwan, A.G. Fractional X-shape Controllable Multi-scroll Attractor with Parameter Effect and FPGA Automatic Design Tool software. Chaos Solitan Fractals 2019, 126, 292-307. [CrossRef]

12. Sayed, W.S.; Ismail, S.M.; Said, L.A.; Radwan, A.G. On the fractional order generalized discrete maps. In Mathematical Techniques of Fractional Order Systems; Elsevier: Amsterdam, The Netherlands, 2018; pp. 375-408.

13. Ismail, S.M.; Radwan, A.G.; Madian, A.H.; Abu-ElYazeed, M.F. Comparative study of fractional filters for alzheimer disease detection on MRI images. In Proceedings of the International Conference on Telecommunications and Signal Processing (TSP), Vienna, Austria, 27-29 June 2016; pp. 720-723.

14. Abdel-Gawad, A.H.; Said, L.A.; Radwan, A.G. Optimized Edge Detection Technique for Brain Tumor Detection in MR Images. IEEE Access 2020, 8, 136243-136259. [CrossRef]

15. $\mathrm{Pu}, \mathrm{Y}$. Fractional calculus approach to texture of digital image. In Proceedings of the IEEE International Conference on Signal Processing (ICSP), Guilin, China, 16-20 November 2006; pp. 1002-1006.

16. Chen, J.; Huang, C.; Du, Y.; Lin, C. Combining fractional-order edge detection and chaos synchronisation classifier for fingerprint identification. IET Image Process 2014, 8, 354-362. [CrossRef]

17. Chi, C.; Gao, F. Palm Print Edge Extraction Using Fractional Differential Algorithm. J. Appl. Math. 2014, 2014. [CrossRef]

18. Pu, Y.-F. Fractional differential analysis for texture of digital image. J. Algorithms Comput. Technol. 2007, 1, 357-380. [CrossRef]

19. Jia, H.; Pu, Y. Fractional calculus method for enhancing digital image of bank slip. In Proceedings of the Congress on Image and Signal Processing, Sanya, China, 27-30 May 2008; Volume 3, pp. 326-330.

20. Marazzato, R.; Sparavigna, A.C. Astronomical image processing based on fractional calculus: The AstroFracTool. arXiv 2009, arXiv:0910.4637.

21. Dong, F.; Chen, Y. A Fractional-order derivative based variational framework for image denoising. Inverse Problems Imaging 2016, 10, 27-50. [CrossRef] 
22. Hemalatha, S.; Anouncia, S.M. Anouncia, S.M. A computational model for texture analysis in images with fractional differential filter for texture detection. In Biometrics: Concepts, Methodologies, Tools, and Applications; IGI Global: Vellore, India, 2017; pp. 309-332.

23. Ghanbari, B.; Atangana, A. A new application of fractional Atangana-Baleanu derivatives: Designing ABC-fractional masks in image processing. Phys. A Stat. Mech. Its Appl. 2019. [CrossRef]

24. Telke, C.; Beitelschmidt, M. Edge detection based on fractional order differentiation and its application to railway track images. Pamm 2015, 15, 671-672. [CrossRef]

25. He, C.; Ye, Y.Q.; Jiang, B.; Zhou, X. A novel edge detection method based on fractional-order calculus mask. Acta Autom. Sin. 2012, 38, 776-787. [CrossRef]

26. Wang, W.X.; Li, W.S.; Yu, X. Fractional differential algorithms for rock fracture images. Imaging Sci. J. 2012, 60, 103-111. [CrossRef]

27. Amoako-Yirenkyi, P.; Appati, J.K.; Dontwi, I.K. A new construction of a fractional derivative mask for image edge analysis based on Riemann-Liouville fractional derivative. Adv. Differ. Equ. 2016, 2016, 238. [CrossRef]

28. Daou, R.A.Z.; El Samarani, F.; Yaacoub, C.; Moreau, X. Fractional Derivatives for Edge Detection: Application to Road Obstacles In Smart Cities Performability, Cognition, E Security; Springer: Cham, Switzerland, 2020; pp. 115-137.

29. Tian, D.; Wu, J.F.; Yang, Y.J. A fractional-order Sobel operator for medical image structure feature extraction. In Advanced Materials Research; Trans Tech Publications Ltd.: Bäch, Switzerland, 2014; Volume 860, pp. 2910-2913.

30. Chen, Q.; Huang, G.; Men, T.; Qin, H.; Wang, M. Fractional differential algorithm for texture and contrast enhancement. In Proceedings of the Eighth International Conference on Digital Image Processing (ICDIP), Chengdu, China, 20-22 May 2016; International Society for Optics and Photonics: Bellingham, WA, USA, 2016; Volume 10033, p. 1003323.

31. Bist, A.; Sondhi, S.G. Fractional Order Approach for Edge Detection of Low Contrast Images. Master's Thesis, Thapar Institute of Engineering and Technology, Punjab, India, 2017.

32. Nandal, A.; Gamboa-Rosales, H.; Dhaka, A.; Celaya-Padilla, J.M.; Galvan-Tejada, J.I.; Galvan-Tejada, C.E.; Martinez-Ruiz, F.J.; Guzman-Valdivia, C. Image edge detection using fractional calculus with feature and contrast enhancement. Circuits Syst. Signal Process. 2018, 37, 3946-3972. [CrossRef]

33. Tian, D.; Wu, J.; Yang, Y. A Fractional-order Edge Detection Operator for Medical Image Structure Feature Extraction. In Proceedings of the 26th IEEE Chinese Control and Decision Conference, Changsha, China, 31 May-2 June 2014; pp. 5173-5176.

34. Bento, T.; Valério, D.; Teodoro, P.; Martins, J. Fractional Order Image Processing of Medical Images. J. Appl. Nonlinear Dyn. 2017, 6, 181-191. [CrossRef]

35. ElAraby, W.S.; Madian, A.H.; Ashour, M.A.; Farag, I.; Nassef, M. Fractional edge detection based on genetic algorithm. In Proceedings of the 2017 29th International Conference on Microelectronics (ICM), Hamra, Beirut, 10-13 December 2017; pp. 1-4.

36. John, R.; Kunju, N. Detection of Alzhemier's Disease Using Fractional Edge Detection. Glob. J. Technol. Optim. 2019, 9. [CrossRef]

37. Lavín-Delgado, J.E.; Solís-Pérez, J.E.; Gómez-Aguilar, J.F.; Escobar-Jiménez, R.F. A New Fractional-Order Mask for Image Edge Detection Based on Caputo-Fabrizio Fractional-Order Derivative without Singular Kernel. Circuits Syst. Signal Process. 2019, 39, 1419-1448. [CrossRef]

38. Pan, X.; Zhu, J.; Yu, H.; Chen, L.; Liu, Y.; Li, L. Robust corner detection with fractional calculus for magnetic resonance imaging. Biomed. Signal Process. Control 2021, 63, 102112. [CrossRef]

39. Kaur, K.; Jindal, N.; Singh, K. Fractional Fourier Transform based Riesz fractional derivative approach for edge detection and its application in image enhancement. Signal Process. 2021, 180, 107852. [CrossRef]

40. Lv, H.; Zhang, F.; Wang, R. Robust Active Contour Model Using Patch-Based Signed Pressure Force and Optimized FractionalOrder Edge. IEEE Access 2021, 9, 8771-8785. [CrossRef]

41. Samko, S.G.; Kilbas, A.A.; Marichev, O.I. Fractional Integrals and Derivatives; Gordon and Breach Science Publishers: Yverdon, Switzerland, 1993; Volume 1993.

42. The Berkley Segmentation Dataset and Benchmark. 2007. Available online: https://www2.eecs.berkeley.edu/Research/Projects/ CS/vision/grouping/segbench/ (accessed on 5 March 2021).

43. Mansour, R.F. Evolutionary computing enriched computer-aided diagnosis system for diabetic retinopathy: A survey. IEEE Rev. Biomed. Eng. 2017, 10, 334-349. [CrossRef] [PubMed]

44. Fraz, M.M.; Remagnino, P.; Hoppe, A.; Uyyanonvara, B.; Rudnicka, A.R.; Owen, C.G.; Barman, S.A. Blood vessel segmentation methodologies in retinal images-a survey. Comput. Methods Programs Biomed. 2012, 108, 407-433. [CrossRef] [PubMed]

45. Ismail, S.M. Fractional-Order Image Segmentation for Security Surveillance. In Proceedings of the 32nd International Conference on Microelectronics (ICM), Aqaba, Jordan, 14-16 December 2020.

46. Yazid, H.; Arof, H.; Mokhtar, N. Edge sharpening for diabetic retinopathy detection. In Proceedings of the IEEE Conference on Cybernetics and Intelligent Systems (CIS), Singapore, 28-30 June 2010; pp. 41-44.

47. Sivakamasundari, J.; Kavitha, G.; Natarajan, V.; Ramakrishnan, S. Proposal of a Content Based retinal Image Retrieval system using Kirsch template based edge detection. In Proceedings of the International Conference in Informatics, Electronics \& Vision (ICIEV), Dhaka, Bangladesh, 23-24 May 2014; pp. 1-5.

48. Dutta, M.K.; Srivastava, K.; Ganguly, S.; Parthasarathi, M.; Burget, R.; Prinosil, J. Exudates detection in digital fundus image using edge based method \& strategic thresholding. In Proceedings of the 38th International Conference On Telecommunications and Signal Processing (TSP), Prague, Czech Republic, 9-11 July 2015; pp. 748-752. 
49. Tasgaonkar, M.; Khambete, M. Using differential morphological profiles for microaneurysm detection in diabetic retinal fundus images. In Proceedings of the International Conference on Signal and Information Processing (IConSIP), Nanded, India, 6-8 October 2016; pp. 1-5.

50. Hoover, A. Structured Analysis of the Retina. 2018. Available online: http://www.ces.clemson.edu/-ahoover/stare (accessed on 5 March 2021). 